\title{
Distribution of the Chemical Composition of Block and Graft Copolymers Assuming Gamma Distribution of Molecular Weights of the Precursor Blocks
}

\author{
Jaroslav STEJSKAL and Pavel KRATOChvíL \\ Institute of Macromolecular Chemistry, Czechoslovak Academy of Sciences, \\ 16206 Prague 6, Czechoslovakia
}

(Received February 16, 1982)

\begin{abstract}
Various models of coupling of precursor blocks leading to the formation of block and/or graft copolymers are defined. Distribution functions of chemical composition, and in some cases also of molecular weights of various types of copolymers have been derived in an analytical form for a case in which precursor blocks obey the Schulz-Zimm (gamma) molecular weight distribution, and the coupling of blocks has a random character. The effect of polydispersity of precursors, simplifying approximations, and certain other factors on the form of distribution functions of the chemical composition of copolymers is discussed. Assumption of another type of molecular weight distribution of precursors does not give qualitatively different results.

KEY WORDS Block and Graft Copolymers / Distribution of Chemical

Composition / Molecular Weight Distribution / Chemical Heterogeneity of Copolymers / Heterogeneity Parameters $P$ and $Q /$ Schulz-Zimm Distribution of Molecular Weights /
\end{abstract}

The chemical heterogeneity of copolymers is a phenomenon inseparably linked with their existence. As has been shown in a basic study by Kotaka et al., ${ }^{1}$ the chemical heterogeneity of block and graft copolymers is sometimes pronounced, although they are formed by joining precursor blocks with a low polydispersity in molecular weight. In the extensive literature dealing with the preparation and investigation of copolymers, the chemical heterogeneity of the individual copolymer species (e.g., diblocks, triblocks, etc.) is not mentioned at all, or is regarded as negligible. In many real cases such approximation appears to be unjustified and may possibly lead to a distorted interpretation of experimental data.

The chemical heterogeneity and molecular weight polydispersity of copolymers may be described by distribution functions of chemical composition and molecular weights or, less adequately, but also with less experimental work involved, in terms of statistical moments of these functions. In principle, distribution functions are amenable to experimental determination after the copolymer has been fractionated by a method which separates a chemically heterogeneous and polydisperse sample with respect either to the chemical composition of the individual macromolecules or to their molecular weight. The methods which can be considered for this purpose are mainly chromatographic (thin-layer chromatography, ${ }^{2,3}$ gel-permeation chromatography, ${ }^{1,4,5}$ etc.), and fractionation (various fractionation procedures, ${ }^{6}$ cross-fractionation $\left.{ }^{3,7}\right)$. Experimentally obtained estimates of the chemical composition and molecular weight distributions can then be compared with distribution functions calculated for models, thus checking their adequacy. The chemical heterogeneity parameters $P$ and $Q$ have a similar importance and use; they are statistical moments of distribution functions, and can in principle be determined by light scattering. ${ }^{8-11}$

Distribution functions of the chemical composition of block and graft copolymers are discussed in the literature mostly at a rather general level. If they are to be applied to a particular case, simplifying assumptions must be introduced (such as, e.g., approximation of equal blocks ${ }^{1,4}$ ), and a certain form of the molecular weight distribution of precursor blocks must also be assumed. For this pur- 
pose, the Schulz-Zimm distribution of molecular weights was used in several cases (numerical calculation of the distribution functions of chemical composition $^{1}$ and explicit analytical forms for the diblock ${ }^{11}$ and radial teleblock copolymers ${ }^{5}$ ). In most cases, this distribution function is a good approximation of the true molecular weight distributions of precursors blocks.

In this study, we want to show what conclusions about the distribution functions of chemical composition and molecular weights of copolymers can be obtained by assuming the Schulz-Zimm (gamma) molecular weight distribution of precursor blocks. Accordingly, we derive analytical expressions for the distribution functions of the chemical composition for copolymers of various types. Using these expressions, one can avoid multiple numerical integration, which is often very computer-time consuming, without making the simplifying approximation of equal blocks, which serves the same purpose.

\section{Distribution Functions and Their Moments}

Let us consider copolymer molecules consisting of two parts, each formed by blocks of repeating constitutional units of either the A or B type. The two-dimensional differential number distribution function $N_{\mathrm{C}}\left(M_{\mathrm{A}}, M_{\mathrm{B}}\right)$ perfectly describes the relative amount of molecules with given molecular weights, $M_{\mathrm{A}}$ and $M_{\mathrm{B}}$, of both components. Although the function is often used in the literature in the form, ${ }^{12,13}$ in many respects it is preferable to use as variables the molecular weight of the copolymer macromolecule, $M=M_{\mathrm{A}}+M_{\mathrm{B}}$, and its chemical composition, $x=M_{\mathrm{A}} /\left(M_{\mathrm{A}}+M_{\mathrm{B}}\right)$, given by the weight fraction of component $\mathrm{A}$. Both copolymer parts A and B, may consist of several precursor blocks. Replacement of the variables leads to an analogous distribution function of molecular weights, $M$, and chemical composition, $x$,

$$
N(M, x)=M N_{\mathrm{C}}[x M,(1-x) M]
$$

The corresponding weight distribution function is given by

$$
W(M, x)=\frac{M}{M_{n}} N(M, x)
$$

where $M_{\mathrm{n}}$ is the number average molecular weight of the copolymer. From here on, the term distribu- tion function will always mean the differential distribution function.

Marginal weight distribution functions of molecular weights and chemical composition are given by integrals

$$
\begin{aligned}
& W(M)=\int_{0}^{1} W(M, x) \mathrm{d} x \\
& W(x)=\int_{0}^{\infty} W(M, x) \mathrm{d} M
\end{aligned}
$$

Analogous relations hold for marginal number distribution functions.

In practice, the following statistical moments of the distribution function $W(M, x)$ are of importance: the weight average chemical composition, $x_{w}$, and weight average molecular weight, $M_{w}$, and the parameters of chemical heterogeneity $P$ and $Q$ defined by

$$
\begin{aligned}
& x_{w}=\iint_{x M} x W(M, x) \mathrm{d} M \mathrm{~d} x \\
& M_{w}=\iint_{x M} M W(M, x) \mathrm{d} M \mathrm{~d} x \\
& P=\iint_{x M}\left(x-x_{w}\right) M W(M, x) \mathrm{d} M \mathrm{~d} x \\
& Q=\iint_{x M}\left(x-x_{w}\right)^{2} M W(M, x) \mathrm{d} M \mathrm{~d} x
\end{aligned}
$$

The parameter $P$ is a measure of mutual correlation of the distribution of molecular weights and chemical composition, the parameter $Q$ characterizes the extent of chemical heterogeneity of the copolymer. The moments of the number distribution function $N(M, x)$, e.g., the number average chemical composition, $x_{n}$, and number average molecular weight, $M_{n}$, are defined similarly:

$$
\begin{aligned}
& x_{n}=\iint_{x M} x N(M, x) \mathrm{d} M \mathrm{~d} x \\
& M_{n}=\iint_{x M} M N(M, x) \mathrm{d} M \mathrm{~d} x
\end{aligned}
$$

We shall now derive the distribution functions of molecular weights and chemical composition for various types of copolymers, assuming that the precursor blocks have the gamma distribution of molecular weights. Some properties of this distribution are briefly presented below. 


\section{Gamma Distribution of Molecular Weights}

The gamma (Schulz-Zimm) molecular weight distribution is described by a two-parameter distribution function

$$
\Gamma(M ; y, h)=\frac{h^{y}}{\Gamma(y)} M^{y-1} \exp (-h M)
$$

with the positive parameters $y$ and $h ; \Gamma(y)$ is the $\Gamma$ function.

If the molecular weight number distribution function of the polymer is given by eq 6 , the corresponding weight distribution function has a similar form:

$$
\begin{gathered}
N(M)=\Gamma(M ; y, h) \\
W(M)=\Gamma(M ; y+1, h)
\end{gathered}
$$

The parameters of the distribution functions are related with the molecular weight averages through

$$
M_{n}=\frac{y}{h}, \quad M_{w}=\frac{y+1}{h}
$$

and thus the parameter $y$ reflects the polydispersity in molecular weight,

$$
y=\frac{1}{\frac{M_{w}}{M_{n}}-1}
$$

\section{MODELS OF COPOLYMER FORMATION}

Let us have two sets of macromolecules, one of type A and the other of type B. The molecular weight distributions of these macromolecules (precursor blocks) are described by the differential number and/or weight distribution functions $N_{\mathrm{A}}^{*}\left(M_{\mathrm{A}}^{*}\right), W_{\mathrm{A}}^{*}\left(M_{\mathrm{A}}^{*}\right)$ and $N_{\mathrm{B}}^{*}\left(M_{\mathrm{B}}^{*}\right), W_{\mathrm{B}}^{*}\left(M_{\mathrm{B}}^{*}\right)$. The possibilities will be examined which may arise if the copolymer macromolecule is created by suitably coupling various numbers of precursor blocks A and B. The term precursor blocks denotes chains which, by combining, yield a block or graft copolymer. In the former case, block $\mathrm{A}$ is the basic block and block B, the attached one: in the latter, block of type A forms the backbone and blocks B form grafts in those cases where such distinction is useful.

If it is assumed that the molecular weight distributions of polymers $\mathrm{A}$ and $\mathrm{B}$ are mutually independent and that the coupling of presursor macromolecules is random, three basic types of coupling can be distinguished (models I-III). These are demonstrated using a simple example of mutual coupling of one block $A$ and one block B each time. By generalizing this approach, more complex structures consisting of more than two blocks can also be described.

\section{Model I}

Each of the macromolecules A and B bears one constitutional unit capable of coupling with a macromolecule of different type (Figure 1a). The number fraction of macromolecules containing part A with the molecular weight $M_{\mathrm{A}}$ and part B with the molecular weight $M_{\mathrm{B}}$ is given by the number fractions of precursor molecules with these molecular weights,

$$
\begin{aligned}
& N_{\mathrm{C}}\left(M_{\mathrm{A}}, M_{\mathrm{B}}\right) \mathrm{d} M_{\mathrm{A}} \mathrm{d} M_{\mathrm{B}} \\
& =N_{\mathrm{A}}^{*}\left(M_{\mathrm{A}}^{*}\right) N_{\mathrm{B}}^{*}\left(M_{\mathrm{B}}^{*}\right) \mathrm{d} M_{\mathrm{A}}^{*} \mathrm{~d} M_{\mathrm{B}}^{*}
\end{aligned}
$$

where $N_{\mathrm{C}}\left(M_{\mathrm{A}}, M_{\mathrm{B}}\right)$ is a two-dimensional differential number distribution function of molecular weights $M_{\mathrm{A}}$ and $M_{\mathrm{B}}$ of blocks which are part of the copolymer $C$. The molecular weight distributions of reacted and unreacted precursors $\mathrm{A}$ and $\mathrm{B}$ are identical and independent of the conversion of
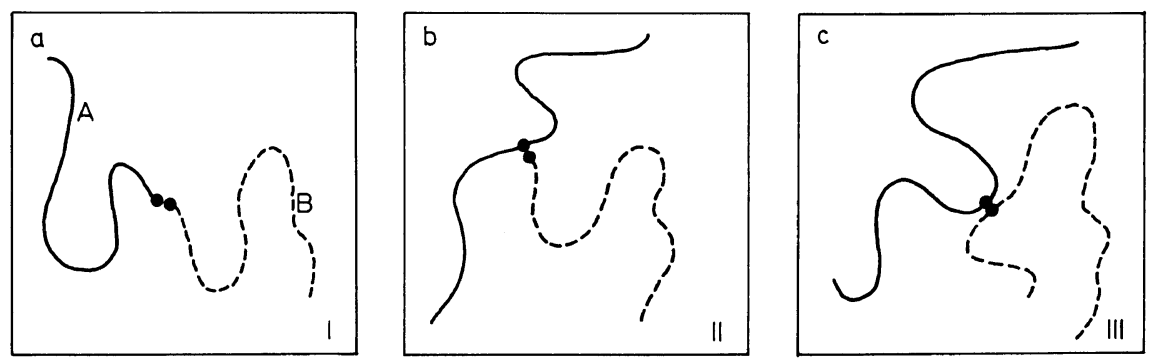

Figure 1. Three basic types of copolymer macromolecule formed by coupling one block $\mathrm{A}$ with one block B. 
precursors into the copolymer. This model can, e.g., be used in the description of a diblock formed in block copolymerization.

\section{Model II}

Let it be assumed that, in the polymer A, each of its repeating constitutional units is able to become a coupling unit and that the polymer block B may, similarly to the preceding case, be coupled by a single (e.g., end) constitutional unit (Figure 1b). This model corresponds to the graft copolymer with the backbone A to which one graft B has been attached. The probability that macromolecules of type A with molecular weight $M_{\mathrm{A}}^{*}$ will become the backbone of a copolymer molecule is proportional to their molecular weight (i.e., to the number of potential coupling units) and to their number fraction, i.e., altogether to their weight fraction in the parent polymer $\mathrm{A}$,

$$
\begin{aligned}
& N_{\mathrm{C}}\left(M_{\mathrm{A}}, M_{\mathrm{B}}\right) \mathrm{d} M_{\mathrm{A}} \mathrm{d} M_{\mathrm{B}} \\
& \quad=W_{\mathrm{A}}^{*}\left(M_{\mathrm{A}}^{*}\right) N_{\mathrm{B}}^{*}\left(M_{\mathrm{B}}^{*}\right) \mathrm{d} M_{\mathrm{A}}^{*} \mathrm{~d} M_{\mathrm{B}}^{*}
\end{aligned}
$$

In this case, the stituation is more complicated than in the preceding model. Since it is preferentially macromolecules A with the higher molecular weight which become part of the copolymer, the molecular weight distribution of unreacted backbones will vary with increasing conversion of precursor $\mathrm{A}$ into the copolymer. This is the basic difference between the two models. Hence, eq $9 b$ is valid in the limiting case of zero conversion, or in each moment of the coupling reaction (the distribution functions in eq $9 \mathrm{~b}$ are then instantaneous distribution functions). Like the preceding case, the molecular weight distributions of grafts in the copolymer and of the unattached grafts are in a simple relation (not necessarily identical in real cases, this depending on, e.g., the termination reaction of the grafts) and are conversion-independent. In practice, this model is approached by, e.g., preparation of a graft copolymer by irradiation of the polymer $\mathrm{A}$ dissolved in the monomer $\mathrm{B}$, when radicals formed on the backbone $A$ initiate the growth of the polymer blocks B, and the propagating radicals are terminated by disproportionation.

\section{Model III}

Each repeating constitutional unit of any of the macromolecules $\mathrm{A}$ and $\mathrm{B}$ possesses the ability to become a coupling unit (Figure 1c). The number of copolymer macromolecules which will contain parts A and B with the molecular weights $M_{\mathrm{A}}$ and $M_{\mathrm{B}}$ will therefore be proportional to the weight content of precursor blocks with the particular molecular weights,

$$
\begin{aligned}
& N_{\mathrm{C}}\left(M_{\mathrm{A}}, M_{\mathrm{B}}\right) \mathrm{d} M_{\mathrm{A}} \mathrm{d} M_{\mathrm{B}} \\
& \quad=W_{\mathrm{A}}^{*}\left(M_{\mathrm{A}}^{*}\right) W_{\mathrm{B}}^{*}\left(M_{\mathrm{B}}^{*}\right) \mathrm{d} M_{\mathrm{A}}^{*} \mathrm{~d} M_{\mathrm{B}}^{*}
\end{aligned}
$$

What in the case of the preceding model is valid for the backbone block A, holds here for both components $\mathrm{A}$ and $\mathrm{B}$. For instance, copolymers arising by irradiation of a mixture of two homopolymers in solution may in the first approximation be described by a model of this type, if no splitting of the main chains takes place.

If the macromolecule contains more than one, but not all constitutional units capable of coupling with the other macromolecule (e.g., diions in block copolymerization, reactive groups introduced into the copolymer chain by statistical copolymerization with a suitable monomer, or reactive groups due to the polymer-analogous reaction, etc.), the following holds: if each macromolecule $\mathrm{A}$ bears the same number of reactive units irrespective of its molecular weight, then the probability of formation of a copolymer with the molecular weight of component A equal just to $M_{\mathrm{A}}$ is proportional to the number distribution function, $N_{\mathrm{A}}^{*}\left(M_{\mathrm{A}}^{*}\right) \mathrm{d} M_{\mathrm{A}}^{*}$. When the number of reactive groups or potential coupling units in the macromolecule is directly proportional to its molecular weight, such probability is proportional to the weight distribution function $W_{\mathrm{A}}^{*}\left(M_{\mathrm{A}}^{*}\right) \mathrm{d} M_{\mathrm{A}}^{*}$. The concept of polymer segment has also been introduced in the literature, ${ }^{4}$ referring to a part of the macromolecule bearing just one potential coupling unit.

Cases of coupling of more than two polymer blocks may be solved by a multiple application of the above models, i.e., by a hypothetical coupling of the required number of blocks of type $A$ to one part of a copolymer molecule and of the given number of blocks B to another part followed by the coupling of these two parts into a resulting copolymer macromolecule, while respecting differences of the individual models.

In order to render the relations more graphic, the differentials $\mathrm{d} M_{\mathrm{A}}$ and $\mathrm{d} M_{\mathrm{B}}$ are henceforward omitted. Now, some types of copolymers will be exam- 
ined in greater detail.

\section{APPLICATION OF MODELS}

\section{Block Copolymers}

A block copolymer consisting of macromolecules formed by joining $a$ precursor blocks of the type A and $b$ blocks of the type $\mathbf{B}$ is considered (Figures 2ac). Here again, all quantities related to the properties of precursor blocks are marked with asterisks; symbols without asterisks are assigned to whole copolymer parts $\mathrm{A}$ and $\mathrm{B}$, generally consisting of several blocks. Thus, $N_{\mathrm{A}}^{*}\left(M_{\mathrm{A}}^{*}\right)$ is the number distribution function of molecular weights $M_{\mathrm{A}}^{*}$ of precursor blocks of the type A. From the formal viewpoint, distribution functions for more complicated structures are advantageously denoted as conditioned distribution functions, i.e., e.g., $N_{\mathrm{A}}\left(M_{\mathrm{A}} \mid a\right)$ is the number distribution function of molecular weights $M_{\mathrm{A}}$ of the copolymer part $\mathrm{A}$ on condition that the latter consists just of $a$ blocks, and the like.

Let us now search for an expression for the number distribution function of molecular weights of copolymer macromolecules, $N_{\mathrm{C}}\left(M_{\mathrm{A}}, M_{\mathrm{B}} \mid a, b\right)$, which contain part A having the total molecular weight $M_{\mathrm{A}}=M_{\mathrm{A} 1}^{*}+M_{\mathrm{A} 2}^{*}+\cdots+M_{\mathrm{Aa}}^{*}$, and similarly part B having the total molecular weight $M_{\mathrm{B}}=M_{\mathrm{B} 1}^{*}+M_{\mathrm{B} 2}^{*}+\cdots+M_{\mathrm{Bb}}^{*}$. Sums in the definitions of $M_{\mathrm{A}}$ and $M_{\mathrm{B}}$ represent an arbitrary combination of polymer blocks of the given type which form a total having the molecular weight $M_{\mathrm{A}}$ or $M_{\mathrm{B}}$, respectively.

First, the copolymer part A alone is considered. It is assumed that $a-1$ blocks A have been linked to form a unit having the molecular weight $M_{\mathrm{A}}-M_{\mathrm{Aa}}^{*}=M_{\mathrm{A} 1}^{*}+M_{\mathrm{A} 2}^{*}+\cdots+M_{\mathrm{A}(\mathrm{a}-1)}^{*}$ described by the distribution function $N_{\mathrm{A}}\left(M_{\mathrm{A}}-M_{\mathrm{Aa}}^{*} \mid a-1\right)$. To this unit, another $a$-th block is attached according to model I having the molecular weight $M_{\mathrm{Aa}}^{*}(c f$. eq 9a). The number distribution function of molecular weights of the copolymer part $\mathrm{A}$ is then given by

$$
\begin{aligned}
& N_{\mathrm{A}}\left(M_{\mathrm{A}} \mid a\right) \\
& \quad=\int_{M_{\mathrm{A} a}^{*}<M_{\mathrm{A}}} N_{\mathrm{A}}^{*}\left(M_{\mathrm{A} a}^{*}\right) N_{\mathrm{A}}\left(M_{\mathrm{A}}-M_{\mathrm{A} a}^{*} \mid a-1\right) \mathrm{d} M_{\mathrm{A} a}^{*}
\end{aligned}
$$

because one must bear in mind all possible com-
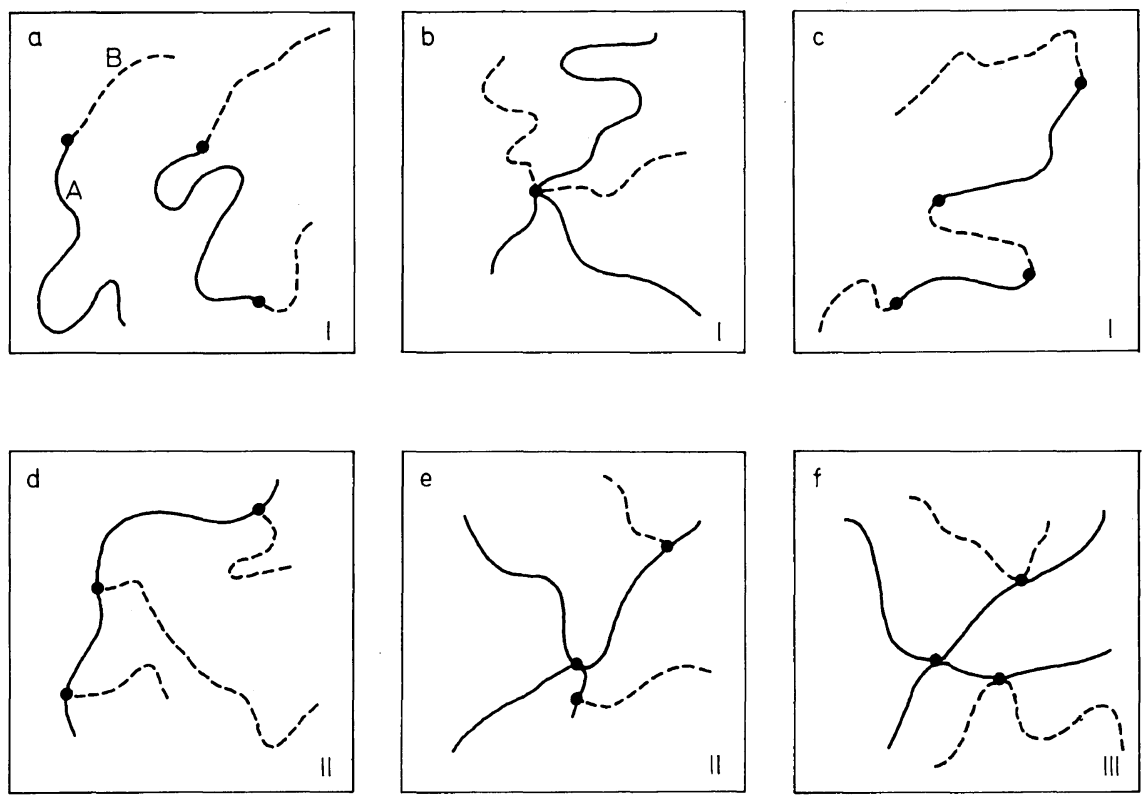

Figure 2. Copolymer structure which can be described by models I through III: (a) block copolymers (diblock and triblock); (b) radial teleblock copolymer; (c) sequential copolymer; (d) graft copolymer with one backbone; $(\mathrm{e}, \mathrm{f})$ graft copolymers with more backbones. 
binations of molecular weights $M_{\mathrm{Aa}}^{*}$ and $\left(M_{\mathrm{A}}-M_{\mathrm{Aa}}^{*}\right)$ which in their sum give $M_{\mathrm{A}}$.

Equation 10 is a recurrent relation, because the distribution function of molecular weights of a more complicated structure is described in terms of the distribution function used for a simpler structure. It can gradually be expanded into an $a$-fold integral, the solution of which is generally difficult. Similar relations hold for the copolymer part B.

If the precursor blocks have the $\Gamma$-distribution of molecular weights

$$
\begin{aligned}
& N_{\mathrm{A}}^{*}\left(M_{\mathrm{A}}^{*}\right)=\Gamma\left(M_{\mathrm{A}}^{*} ; y_{\mathrm{A}}^{*}, h_{\mathrm{A}}^{*}\right) \\
& N_{\mathrm{B}}^{*}\left(M_{\mathrm{B}}^{*}\right)=\Gamma\left(M_{\mathrm{B}}^{*} ; y_{\mathrm{B}}^{*}, h_{\mathrm{B}}^{*}\right)
\end{aligned}
$$

then, using eq 10 , it can be shown that both copolymer parts again exhibit the $\Gamma$-distribution of molecular weights (Appendix A),

$$
\begin{aligned}
& N_{\mathrm{A}}\left(M_{\mathrm{A}} \mid a\right)=\Gamma\left(M_{\mathrm{A}} ; a y_{\mathrm{A}}^{*}, h_{\mathrm{A}}^{*}\right) \\
& N_{\mathrm{B}}\left(M_{\mathrm{B}} \mid b\right)=\Gamma\left(M_{\mathrm{B}} ; b y_{\mathrm{B}}^{*}, h_{\mathrm{B}}^{*}\right)
\end{aligned}
$$

The parameters of these distributions are related in a simple way to analogous parameters of molecular weight distributions of the precursors, $y_{\mathrm{A}}=$ $a y_{\mathrm{A}}^{*}, y_{\mathrm{B}}=b y_{\mathrm{B}}^{*} ; h_{\mathrm{A}}=h_{\mathrm{A}}^{*}$ and $h_{\mathrm{B}}=h_{\mathrm{B}}^{*}$ remain unchanged (Table I). According to eq 8 , the higher value of the parameter $y$ reflects a lower polydispersity index. Hence, the molecular weight distributions of copolymer parts are always narrower than the initial distributions of precursors, if a copolymer part contains more than a single block. A similar finding is well known in, e.g., the kinetics of radical polymerization, where the molecular weight distribution for termination by recombination of polymer radicals (i.e. by coupling two macroradicals each time)

\begin{tabular}{|c|c|c|c|}
\hline \multirow{2}{*}{ Parameter } & \multicolumn{3}{|c|}{$\begin{array}{c}\text { Block copolymer (model I) } \\
\text { with } a \text { blocks of type A and } b \text { blocks of type B }\end{array}$} \\
\hline & \multicolumn{3}{|c|}{ Approximation $^{\mathrm{a}}$} \\
\hline$y_{\mathrm{A}}$ & $a y_{\mathrm{A}}^{*}$ & \multicolumn{2}{|c|}{$y_{\mathrm{A}}^{*}$} \\
\hline$y_{\mathrm{B}}$ & $b y_{\mathrm{B}}^{*}$ & \multicolumn{2}{|c|}{$\begin{array}{l}y_{\mathrm{B}}^{*} \\
h^{*} / a\end{array}$} \\
\hline$h_{\mathrm{A}}$ & $h_{\mathrm{A}}^{*}$ & & \\
\hline \multirow[t]{3}{*}{$h_{\mathrm{B}}$} & $h_{\mathrm{B}}^{*}$ & \multicolumn{2}{|c|}{$h_{\mathbf{B}}^{*} / b$} \\
\hline & \multicolumn{3}{|c|}{$\begin{array}{c}\text { Graft copolymer (model II) } \\
\text { with one backbone of type A and } m \text { grafts of type B }\end{array}$} \\
\hline & Zero conversion & Nonzero conversion & Approximation $^{\mathrm{a}}$ \\
\hline \multirow{6}{*}{$\begin{array}{l}y_{\mathrm{A}} \\
y_{\mathrm{B}} \\
h_{\mathrm{A}} \\
h_{\mathrm{B}}\end{array}$} & $y_{\mathrm{A}}^{*}+m$ & $y_{\mathrm{A}}^{*}+m$ & $y_{\mathrm{A}}^{*}+m$ \\
\hline & $m y_{\mathrm{B}}^{*}$ & $m y_{\mathrm{B}}^{*}$ & $y_{\mathrm{B}}^{*}$ \\
\hline & $h_{\mathrm{A}}^{*}$ & $h_{\mathrm{A}}^{*}+q$ & $h_{\mathrm{A}}^{*}$ \\
\hline & $h_{\mathrm{B}}^{*}$ & $h_{\mathrm{B}}^{*}$ & $h_{\mathbf{B}}^{*} / m$ \\
\hline & \multicolumn{3}{|c|}{$\begin{array}{l}\text { Grafted copolymer (zero conversion), with } l \\
\text { backbones of type A and } m \text { grafts of type B }\end{array}$} \\
\hline & Model II & \multicolumn{2}{|c|}{ Model III } \\
\hline$y_{\mathrm{A}}$ & $l y_{\mathrm{A}}^{*}+2(l-1)+m$ & \multicolumn{2}{|c|}{$l y_{\mathrm{A}}^{*}+2(l-1)+m$} \\
\hline & \multirow{2}{*}{$m y_{\mathrm{B}}^{*}$} & \multirow{2}{*}{\multicolumn{2}{|c|}{$m\left(y_{B}^{*}+1\right)$}} \\
\hline$h_{\mathrm{A}}$ & & \multirow{2}{*}{\multicolumn{2}{|c|}{$\begin{array}{l}h_{\mathrm{A}}^{*} \\
h_{\mathrm{B}}^{*}\end{array}$}} \\
\hline$h_{\mathrm{B}}$ & $h_{\mathrm{B}}^{*}$ & & \\
\hline
\end{tabular}

Table I. Parameters $y$ and $h$ of eq 28-39 characterizing individual parts of copolymer and their relationship to parameters $y^{*}$ and $h^{*}$ of $\Gamma$-distribution of molecular weights of precursors given by eq 11

a Approximation of equal blocks ( $c f$. Discussion). 
is narrower than that observed for termination by disproportionation.

Assuming mutually independent distributions of $M_{\mathrm{A}}$ and $M_{\mathrm{B}}$, the two-dimentional molecular weight number distribution function of copolymer parts can be displayed as a product ( $c f$. eq 9a)

$$
\begin{aligned}
& N_{\mathrm{C}}\left(M_{\mathrm{A}}, M_{\mathrm{B}} \mid a, b\right) \\
& \quad=N_{\mathrm{A}}\left(M_{\mathrm{A}} \mid a\right) N_{\mathrm{B}}\left(\dot{M}_{\mathrm{B}} \mid b\right) \\
& \quad=\Gamma\left(M_{\mathrm{A}} ; y_{\mathrm{A}}, h_{\mathrm{A}}\right) \Gamma\left(M_{\mathrm{B}} ; y_{\mathrm{B}}, h_{\mathrm{B}}\right)
\end{aligned}
$$

Further rearrangement of this equation leading to distribution functions of chemical composition are examined below: it is shown that a formally identical equation is also obtained in the case of graft copolymers.

\section{Graft Copolymers}

(a) Limiting Case of Zero Conversion

A more general case is examined of graft copolymer molecules consisting of $l$ blocks of the type A (backbone) and $m$ blocks of the type B (grafts) (Figure 2e). Thus, we also allow for the possibility of connecting more backbones in one copolymer molecule. The molecular weight distribution of backbones $\mathrm{A}$ in such a copolymer is considered first. By analogy with eq 10 , for a unit obtained by joining $l$ precursor blocks $\mathrm{A}$ according to model III, a recurrent relation can be written which corresponds to the attachment of the $l$-th block A having the molecular weight $M_{\mathrm{A} l}^{*}$ to the structure which already contains $l-1$ joined backbone blocks;

$$
\begin{aligned}
& N_{\mathrm{A}}\left(M_{\mathrm{A}} \mid l\right) \\
& \quad=\int_{M_{\mathrm{A} l}^{*}<M_{\mathrm{A}}} W_{\mathrm{A}}^{*}\left(M_{\mathrm{A} l}^{*}\right) W_{\mathrm{A}}\left(M_{\mathrm{A}}-M_{\mathrm{A} l}^{*} \mid l-1\right) \mathrm{d} M_{\mathrm{A} l}^{*}
\end{aligned}
$$

Since the joining of precursor blocks proceeds differently from the formation of block copolymers, weight distribution functions operate in this case, unlike eq 10 ( $c f$. eq 9c). Assuming the $\Gamma$-distribution of molecular weights (eq 11) for the precursor blocks we obtain

$$
N_{\mathrm{A}}\left(M_{\mathrm{A}} \mid l\right)=\Gamma\left(M_{\mathrm{A}} ; l y_{\mathrm{A}}^{*}+2(l-1), h_{\mathrm{A}}^{*}\right)
$$

Unlike eq $12 \mathrm{a}$, an additive term $2(l-1)$ appears in the first parameter of the distribution function. This is due to the fact that, in the calculation of $N_{\mathrm{A}}\left(M_{\mathrm{A}} \mid l\right)$ according to eq 14 , the number distri- bution function $N_{\mathrm{A}}\left(M_{\mathrm{A}}-M_{\mathrm{A}}^{*} \mid l-1\right)$ calculated earlier must be transformed into a weight distribution function, $W_{\mathrm{A}}\left(M_{\mathrm{A}}-M_{\mathrm{A} l}^{*} \mid l-1\right)$, using eq 2 or 7 .

So far the attachment of grafts has not been considered. One should bear in mind that with respect to model II, according to which blocks B (grafts) are attached in the second stage, the molecular weight number distribution of backbones in the copolymer bearing $m$ grafts is equal to the molecular weight number distribution of backbones in the copolymer with $m-1$ grafts, and thus,

$$
N_{\mathrm{A}}\left(M_{\mathrm{A}} \mid l, m\right)=W_{\mathrm{A}}\left(M_{\mathrm{A}} \mid l, m-1\right)
$$

where $N_{\mathrm{A}}\left(M_{\mathrm{A}} \mid l, m\right)$ is the number distribution function of molecular weights $M_{\mathrm{A}}$ of backbone units consisting of $l$ blocks of the type A, to which $m$ blocks of the type B have been attached. Assuming the $\Gamma$-distribution of molecular weights for the precursors (eq 11), we obtain from eq 15 and 16

$N_{\mathrm{A}}\left(M_{\mathrm{A}} \mid l, m\right)=\Gamma\left(M_{\mathrm{A}} ; l y_{\mathrm{A}}^{*}+2(l-1)+m, h_{\mathrm{A}}^{*}\right)$

Another additive term $m$ in the first parameter of the distribution function is a result of the repeated application of eq 16 and transformation of the number to weight distribution functions (eqs 2 and 7). The character of the $\Gamma$-distribution of molecular weights is preserved also in this case; for $m=0$, eq 17 reduces to eq 15 .

As in the case of block copolymers, the molecular weight distribution of the graft part of the copolymer consisting of $m$ grafts is independent of $l$ and given by

$$
N_{\mathrm{B}}\left(M_{\mathrm{B}} \mid m\right)=\Gamma\left(M_{\mathrm{B}} ; m y_{\mathrm{B}}^{*}, h_{\mathrm{B}}^{*}\right)
$$

As in the preceding case (eq 13), the resulting twodimensional number distribution function of molecular weights of the copolymer parts can be written as

$$
\begin{aligned}
N_{\mathrm{C}}( & \left.M_{\mathrm{A}}, M_{\mathrm{B}} \mid l, m\right) \\
& =W_{\mathrm{A}}\left(M_{\mathrm{A}} \mid l, m-1\right) N_{\mathrm{B}}\left(M_{\mathrm{B}} \mid m\right) \\
& =\Gamma\left(M_{\mathrm{A}} ; y_{\mathrm{A}}, h_{\mathrm{A}}\right) \Gamma\left(M_{\mathrm{B}} ; y_{\mathrm{B}}, h_{\mathrm{B}}\right)
\end{aligned}
$$

where, of course, the relation between the parameters $y$ and $y^{*}$ differs from that valid for block copolymers (Table I).

A comment may be pertinent: If the grafts were attached according to model III (Fig. 2f), the molecular weight distribution of the graft part of the copolymer would be given by 


$$
N_{\mathrm{B}}\left(M_{\mathrm{B}} \mid m\right)=\Gamma\left(M_{\mathrm{B}} ; m\left(y_{\mathrm{B}}^{*}+1\right), h_{\mathrm{B}}^{*}\right)
$$

(cf. eq 18). Generalization to include other structures, e.g., by involving the possibility of further grafting of already attached grafts, can be effected by an algorithm similar to that used for joining the backbones.

We have called these reasonings the limiting case of zero conversion, as they do not take into account one basic fact, namely, that the molecular weight distribution of unreacted backbones varies with conversion of the backbone precursor molecules into the copolymer. Macromolecules with a higher molecular weight are preferentially incorporated into the copolymer, and their participation in the assembly of unreacted backbone blocks decreases more quickly than that of blocks having a lower molecular weight. It can be expected, therefore, that relations derived for graft copolymers hold only when the probability of grafting (and thus also conversion of a backbone precursor molecule into the copolymer) approaches zero. Only then, changes in the molecular weight distribution of unreacted backbone blocks can be regarded as negligible.

Generalization for nonzero conversions is not quite straight-forward, but it is feasible for some simplified models.

Using as an example a simple graft copolymer with a single backbone block with $m$ graft blocks attached (Figure 2d), we demonstrate how the molecular weight distribution of the individual parts of the copolymer changes, if facts so far neglected are taken into account.

\section{(b) Case of Non-Zero Conversion}

The probability that a backbone having the molecular weight $M_{\mathrm{A}}$ contains $m$ attached grafts is given by the binomial distribution ${ }^{4,13,14}$

$$
P_{m}^{n}=b(m ; n, p)=\left(\begin{array}{c}
n \\
m
\end{array}\right) p^{m}(1-p)^{n-m}
$$

where $p$ is the probability that a backbone segment is grafted. A segment is defined as a part of the backbone bearing just one potential coupling unit ${ }^{4}$; the total number of segments in a backbone, $n$, is thus proportional to the molecular weight of the backbone, $M_{\mathrm{A}}$. We introduce an auxiliary parameter $q$ by the equation $n p=q M_{\mathrm{A}}$. Then, $q$ has the meaning of the probability of grafting of a backbone segment per unit molecular weight of the segment ${ }^{1}$ (if each monomeric unit with the molecular weight $M^{0}$ can become a linking unit, i.e. a graft can be attached to it, $\left.q=p / M^{0}\right)$. For $m \ll n$, the binomial distribution given by eq 21 can be approximated by the Poisson distribution ${ }^{4}$

$$
P_{m}^{n}=p\left(m ; q M_{\mathrm{A}}\right)=\frac{\left(q M_{\mathrm{A}}\right)^{m}}{m !} \exp \left(-q M_{\mathrm{A}}\right)
$$

The following relation also holds:

$$
\begin{aligned}
W_{\mathrm{A}}\left(M_{\mathrm{A}}, m\right) & =W_{\mathrm{A}}\left(M_{\mathrm{A}} \mid m\right) W_{\mathrm{A}}(m) \\
& =P_{m}^{n} W_{\mathrm{A}}^{*}\left(M_{\mathrm{A}}^{*}\right)
\end{aligned}
$$

where $W_{\mathrm{A}}\left(M_{\mathrm{A}}, m\right)$ is the two-dimensional weight distribution function which gives the weight portion of backbones having the molecular weight $M_{\mathrm{A}}$ and at the same time carrying $m$ grafts. $W_{\mathbf{A}}\left(M_{\mathrm{A}} \mid m\right)$ is a unidimensional weight distribution function of molecular weights of backbones carrying just $m$ grafts, and finally, $W_{\mathbf{A}}(m)$ represents the weight fraction of backbones with $m$ grafts.

Suppose the initial distribution of precursor blocks $\mathrm{A}$ is given again by the $\Gamma$-distribution of molecular weights, $W_{\mathrm{A}}^{*}\left(M_{\mathrm{A}}^{*}\right)=\Gamma\left(M_{\mathrm{A}}^{*} ; y_{\mathrm{A}}^{*}+1, h_{\mathrm{A}}^{*}\right)$. Then, the integration of eq 23 over all molecular weights $M_{\mathrm{A}}$ yields the distribution function $W_{\mathrm{A}}(m)$ which assumes the form of the negative binomial distribution,

$$
\begin{aligned}
W_{\mathbf{A}}(m) & =n\left(m ; y_{\mathrm{A}}^{*}+1, \frac{h_{\mathrm{A}}^{*}}{h_{\mathrm{A}}^{*}+q}\right) \\
& =\frac{\Gamma\left(y_{\mathrm{A}}^{*}+m+1\right)}{\Gamma\left(y_{\mathrm{A}}^{*}+m\right) m !}\left[1-\frac{h_{\mathrm{A}}^{*}}{h_{\mathrm{A}}^{*}+q}\right]^{m}\left[\frac{h_{\mathrm{A}}^{*}}{h_{\mathrm{A}}^{*}+q}\right]^{y_{\mathrm{A}}^{*}+1}
\end{aligned}
$$

Similarly, the number distribution function which describes the number fraction of backbones carrying $m$ grafts is also given by the distribution of the type

$$
N_{\mathrm{A}}(m)=n\left(m ; y_{\mathrm{A}}^{*}, \frac{h_{\mathrm{A}}^{*}}{h_{\mathrm{A}}^{*}+q}\right)
$$

The molecular weight distributions of backbones carrying just $m$ grafts are again given by the $\Gamma$ distribution

$$
\begin{aligned}
& W_{\mathrm{A}}\left(M_{\mathrm{A}} \mid m\right)=\Gamma\left(M_{\mathrm{A}} ; y_{\mathrm{A}}^{*}+m+1, h_{\mathrm{A}}^{*}+q\right) \\
& N_{\mathrm{A}}\left(M_{\mathrm{A}} \mid m\right)=\Gamma\left(M_{\mathrm{A}} ; y_{\mathrm{A}}^{*}+m, h_{\mathrm{A}}^{*}+q\right)
\end{aligned}
$$

If one compares the latter equation with the result obtained for the limiting case of zero conversion 
given by eq 17 , where $l=1$, it can be seen that for low degrees of grafting where $h_{\mathrm{A}}^{*} \gg q$, both approaches yield the same result. Both the simple model and this approach predict an identical polydispersity of the backbones, but the limiting case does not predict a change in the molecular weights of ungrafted backbones with increasing conversion, unlike the more rigorous approach.

Now, eq $24 \mathrm{a}$ is briefly recalled. According to it for $m=0$, the weight fraction of ungrafted backbones, $W_{\mathbf{A}}(0)$, is given by

$$
W_{\mathrm{A}}(0)=\left[\frac{h_{\mathrm{A}}^{*}}{h_{\mathrm{A}}^{*}+q}\right]^{\mathrm{y}_{\mathrm{A}}^{*}+1}
$$

In principle, this weight fraction can be determined experimentally. Assuming that this model adequately describes the experiment, the parameter $q$ characterizing the efficiency of grafting can then be calculated. Equation 24 can be expressed directly in terms of $W_{\mathrm{A}}(0)$ after substitution from eq 26.

The number and weight averages of the number of grafts attached to the backbones (including the ungrafted backbones) are given by the expression for the average value of the negative binomial distribution

$$
\begin{aligned}
& m_{n}=\sum_{m} m N_{\mathrm{A}}(m)=M_{n \mathrm{~A}}^{*} q \\
& m_{w}=\sum_{m} m W_{\mathrm{A}}(m)=M_{w \mathrm{~A}}^{*} q
\end{aligned}
$$

The meaning of the first average, $m_{n}$, is obvious. It represents the average number of grafts in copolymer macromolecules, because the number fraction of backbones carrying $m$ grafts, $N_{\mathrm{A}}(m)$, at the same time equals the number fraction of copolymer macromolecules carrying $m$ grafts. This of course does not apply to weight fractions.

\section{Distributions Functions of the Chemical Composition and Molecular Weights of the Copolymer}

In the preceding text, we derived the distribution functions of molecular weights of copolymer parts of block and graft copolymers and demonstrated that, if the precursor blocks had the $\Gamma$-distribution of molecular weights, the molecular weight distributions of whole parts A and B of the copolymer, formed generally by several precursor blocks, also possessed the same character. The twodimensional molecular weight number distribution of copolymer parts can be written as a product of two $\Gamma$-distributions ( $c f$. eq 13 and 19):

$$
\begin{aligned}
& N_{\mathrm{C}}\left(M_{\mathrm{A}}, M_{\mathrm{B}}\right) \\
& \quad=\Gamma\left(M_{\mathrm{A}} ; y_{\mathrm{A}}, h_{\mathrm{A}}\right) \Gamma\left(M_{\mathrm{B}} ; y_{\mathrm{B}}, h_{\mathrm{B}}\right)
\end{aligned}
$$

Relations between parameters of this distribution function and parameters of the distribution functions of molecular weights of precursor blocks for various models of the copolymerization mechanism are summarized in Table I.

By transformation of the variables according to eq 1 and 2, we obtain a two-dimensional differential weight distribution function of molecular weights, $M$, and of the chemical composition, x, of the copolymer

$$
\begin{aligned}
W(M, x)= & \frac{h_{\mathrm{A}}^{y_{\mathrm{A}}+1} h_{\mathrm{B}}^{y_{\mathrm{B}}+1}}{\left(y_{\mathrm{A}} h_{\mathrm{B}}+y_{\mathrm{B}} h_{\mathrm{A}}\right) \Gamma\left(y_{\mathrm{A}}\right) \Gamma\left(y_{\mathrm{B}}\right)} x^{y_{\mathrm{A}}-1}(1-x)^{y_{\mathrm{B}}-1} \\
& \times M^{y_{\mathrm{A}}+y_{\mathrm{B}}} \exp \left[-\left(h_{\mathrm{A}} x+h_{\mathrm{B}}(1-x)\right) M\right]
\end{aligned}
$$

According to eq $3 b$, integration over all molecular weights yields a marginal weight distribution function of chemical composition

$$
\begin{aligned}
W(x)= & \frac{h_{\mathrm{A}}^{y_{\mathrm{A}}+1} h_{\mathrm{B}}^{y_{\mathrm{B}}+1}}{\left(y_{\mathrm{A}} h_{\mathrm{B}}+y_{\mathrm{B}} h_{\mathrm{A}}\right)} \frac{\Gamma\left(y_{\mathrm{A}}+y_{\mathrm{B}}+1\right)}{\Gamma\left(y_{\mathrm{A}}\right) \Gamma\left(y_{\mathrm{B}}\right)} \\
& \times \frac{x^{y_{\mathrm{A}}-1}(1-x)^{y_{\mathrm{B}}-1}}{\left[h_{\mathrm{A}} x+h_{\mathrm{B}}(1-x)\right]^{y_{\mathrm{A}}+y_{\mathrm{B}}+1}}
\end{aligned}
$$

The resulting eq 30 is identical with an analogous equation given by Tanaka et al. ${ }^{11}$ for a diblock copolymer. Here, more generally, all parameters refer to the whole copolymer parts rather than to the precursors. The number and weight distribution functions of chemical composition are related with each other by

$$
W(x)=\frac{h_{\mathrm{A}} h_{\mathrm{B}}\left(y_{\mathrm{A}}+y_{\mathrm{B}}\right)}{\left(y_{\mathrm{A}} h_{\mathrm{B}}+y_{\mathrm{B}} h_{\mathrm{A}}\right)\left(h_{\mathrm{A}} x+h_{\mathrm{B}}(1-x)\right)} N(x)
$$

Analogous marginal distribution functions of mo-. lecular weights cannot generally be expressed in a closed form, but only as a series. Integration of eq 29 according to eq $3 \mathrm{a}$ gives the weight distribution function of molecular weights of the copolymer,

$$
\begin{aligned}
W(M)= & \Gamma\left(M ; y_{\mathrm{A}}+y_{\mathrm{B}}+1, h_{\mathrm{B}}\right)\left[\frac{h_{\mathrm{A}}}{h_{\mathrm{B}}}\right]^{y_{\mathrm{A}}} \frac{h_{\mathrm{A}}\left(y_{\mathrm{A}}+y_{\mathrm{B}}\right)}{\left(y_{\mathrm{A}} h_{\mathrm{B}}+y_{\mathrm{B}} h_{\mathrm{A}}\right)} \\
& \times\left[1+\frac{y_{\mathrm{A}}}{y_{\mathrm{A}}+y_{\mathrm{B}}} \frac{\left(h_{\mathrm{A}}-h_{\mathrm{B}}\right) M}{1 !}\right.
\end{aligned}
$$




$$
\left.+\frac{y_{\mathrm{A}}\left(y_{\mathrm{A}}+1\right)}{\left(y_{\mathrm{A}}+y_{\mathrm{B}}\right)\left(y_{\mathrm{A}}+y_{\mathrm{B}}+1\right)} \frac{\left(h_{\mathrm{A}}-h_{\mathrm{B}}\right)^{2} M^{2}}{2 !}+\cdots\right]
$$

In the particular case, where $h_{\mathrm{A}}=h_{\mathrm{B}}=h$, i.e., if the difference between the molecular weight averages for both parts of the copolymer, A and B, is the same, $M_{w \mathrm{~A}}-M_{n \mathrm{~A}}=M_{w \mathrm{~B}}-M_{n \mathrm{~B}}(c f$. eq 8$)$, the distribution function given by eq 29 is reduced to a simple form

$$
W(\mathbf{M}, x)=\Gamma\left(M ; y_{\mathbf{A}}+y_{\mathbf{B}}+1, h\right) B\left(x ; y_{\mathbf{A}}, y_{\mathbf{B}}\right)
$$

$B\left(x ; y_{\mathrm{A}}, y_{\mathrm{B}}\right)$ is the beta distribution function defined as,

$$
B\left(x ; y_{\mathrm{A}}, y_{\mathrm{B}}\right)=B^{-1}\left(y_{\mathrm{A}}, y_{\mathrm{B}}\right) x^{y_{\mathrm{A}}-1}(1-x)^{y_{\mathrm{B}}-1}
$$

where $B\left(y_{\mathrm{A}}, y_{\mathrm{B}}\right)=\Gamma\left(y_{\mathrm{A}}\right) \Gamma\left(y_{\mathrm{B}}\right) / \Gamma\left(y_{\mathrm{A}}+y_{\mathrm{B}}\right)$ is the beta function.

As can be seen in eq 33, the relation $W(M, x)=$ $W(M) W(x)$ holds, so that in this case the distributions of molecular weights and chemical compositions are independent of each other. Also under these conditions, the parameter $P$ defined by eq $4 c$, which is a measure of the mutual dependence of both distributions, is zero and the number and weight distribution functions of chemical composition are identical, $W(x)=N(x)$, as follows from eq 31 .

Calculation of the molecular weight $M_{w}$, or of $M_{w} / M_{n}$, and of the parameters of chemical heterogeneity $P$ and $Q$ from the definition eqs 4 leads to

$$
\begin{aligned}
\frac{M_{w}}{M_{n}} & =\frac{x_{w}^{2} y_{\mathrm{B}}+y_{\mathrm{A}} y_{\mathrm{B}}+\left(1-x_{w}\right)^{2} y_{\mathrm{A}}}{y_{\mathrm{A}} y_{\mathrm{B}}} \\
\frac{P}{M_{w}} & =\frac{x_{w}\left(1-x_{w}\right)\left[x_{w} y_{\mathrm{B}}-\left(1-x_{w}\right) y_{\mathrm{A}}\right]}{x_{w}^{2} y_{\mathrm{B}}+y_{\mathrm{A}} y_{\mathrm{B}}+\left(1-x_{w}\right)^{2} y_{\mathrm{A}}} \\
\frac{Q}{M_{w}} & =\frac{x_{w}^{2}\left(1-x_{w}^{2}\right)\left(y_{\mathrm{A}}+y_{\mathrm{B}}\right)}{x_{w}^{2} y_{\mathrm{B}}+y_{\mathrm{A}} y_{\mathrm{B}}+\left(1-x_{w}\right)^{2} y_{\mathrm{A}}}
\end{aligned}
$$

These relations have been proved ${ }^{1,10}$ to hold independently of the distribution function $W(M, x)$ and of copolymer structure. Parameters $y_{\mathrm{A}}$ and $y_{\mathrm{B}}$ generally characterize the polydispersities of the individual copolymer parts, $y_{\mathrm{K}}=1 /\left(M_{w \mathrm{~K}} / M_{n \mathrm{~K}}-1\right)$, $K=\mathrm{A}$ or $\mathrm{B}$. The papers referred to give identical equations, differing only in their formal layout.

For precursors with $\Gamma$-distribution of molecular weights, parameters $y_{\mathrm{A}}$ and $y_{\mathrm{B}}$ can be derived from the corresponding precursor characteristics, $y_{\mathrm{A}}^{*}$ and
$y_{\mathrm{B}}^{*}$, using Table I. For these kinds of precursors, two additional relations hold:

$$
\begin{gathered}
x_{w}=\frac{y_{\mathrm{A}} h_{\mathrm{B}}}{y_{\mathrm{A}} h_{\mathrm{B}}+y_{\mathrm{B}} h_{\mathrm{A}}} \\
M_{n}=\frac{y_{\mathrm{A}} h_{\mathrm{B}}+y_{\mathrm{B}} h_{\mathrm{A}}}{h_{\mathrm{A}} h_{\mathrm{B}}}
\end{gathered}
$$

\section{Mixtures of Copolymer Species}

In most cases, synthesis of copolymers leads to a mixture of copolymer macromolecules differing not only in chemical composition and molecular weight, but also in the number of constituent blocks. Below, the term copolymer species is applied to a set of copolymer macromolecules consisting of a given number of precursor blocks A and blocks B. Thus, for instance, in the preparation of a triblock copolymer, the reaction product usually contains, in addition to the required triblocks, also other species, viz., diblocks and homopolymers. Especially, the syntheses of graft copolymers may yield mixtures rich in various copolymer species. In these cases, it is suitable to introduce distribution functions for the copolymer as a whole (if so desired, also including the accompanying homopolymers), which are a weighted sum of the distribution functions of the individual species $s$ appearing in the mixture,

$$
\begin{aligned}
W_{\mathrm{C}}(M, x) & =\sum_{s} W(s) W(M, x \mid s) \\
W_{\mathrm{C}}(x) & =\sum_{s} W(s) W(x \mid s) \\
W_{\mathrm{C}}(M) & =\sum_{s} W(s) W(M \mid s)
\end{aligned}
$$

etc., where the distribution function $W(s)$ describes the weight fractions of the individual species $s$ in the mixture; e.g., $W(M \mid s)$ is the molecular weight distribution of the copolymer species $s$, etc.

The form of the distribution functions $W(s)$ for a given case must be derived from the mechanism of the reaction used in the preparation of the copolymers, or a simplifying model should be chosen. When the reaction mechanism is too complicated or not sufficiently understood. If, e.g., we assume in a simplified version that the graft copolymer consists merely of macromolecules which possess only one backbone and various numbers $m$ of the attached grafts, then the weight distribution of the individual species $W(s)$ can be described by means of the 
distribution $W_{\mathbf{A}}(m)$ given by eq $24 \mathrm{a}$. The distributions of the individual species for various concrete cases have been described in the literature. ${ }^{1,9}$

For parameters of the chemical heterogeneity of a copolymer formed by a mixture of chemically heterogeneous copolymer species, $s$, it can be written that (Appendix B):

$$
\begin{aligned}
& P_{\mathrm{C}}=P_{s}^{(1)}+P_{s}^{(2)} \\
& Q_{\mathrm{C}}=Q_{s}^{(1)}+Q_{s}^{(2)}+2\left[\sum_{s} x_{s} P_{s} W(s)-x_{s}^{(1)} P_{s}^{(1)}\right] \\
& M_{w, \mathrm{C}}=M_{s}^{(1)}, \quad x_{\mathrm{C}}=x_{s}^{(1)}
\end{aligned}
$$

where $A_{s}^{(1)}$ is the weight average of the quantity $A_{s}=$ $P_{s}, Q_{s}, M_{s}$, or $x_{s}$ of the mixture,

$$
A_{s}^{(1)}=\sum_{s} A_{s} W(s)
$$

and $A_{s}^{(2)}$ are parameters of the chemical heterogeneity, $P$ and $Q$, calculated for a similar hypothetical mixture, all the individual components (species) of which are chemically homogeneous.

\section{EXAMPLES AND DISCUSSION}

\section{Distribution Functions of Chemical Composition}

First, some basic properties of the distribution functions of chemical composition and of their parameters are shown using several examples. Again, the basic block or the backbone is referred to as part $\mathrm{A}$ and the attached blocks or grafts as part B, where such distinction is desirable. The compositions are given by the weight fraction of component A.

The required characteristics of the copolymer are obtained by the following procedure:

(a) From the known molecular weights of precursors or parent polymers, the parameters $y^{*}$ and $h^{*}$ of the $\Gamma$-distribution of molecular weights are calculated using eq 8 .

(b) Table I gives the relation between the parameters $y$ and $y^{*}, h$ and $h^{*}$ according to the chosen model.

(c) The required distribution functions of chemical composition and molecular weight are calculated from eq 29-34; the corresponding parameters of chemical heterogeneity of copolymers are calculated by means of eq 36 and 37 .

A diblock, $\mathrm{AB}$, and a triblock, $\mathrm{BAB}$ copolymer are considered, formed according to model I from precursors A and B having in both cases the same $\Gamma$ distribution of molecular weights and $M_{n}^{*}=50,000$, $M_{w}^{*} / M_{n}^{*}=1.2$ (the molecular weight distribution is relatively narrow). According to eq $8, y_{\mathrm{A}}^{*}=y_{\mathrm{B}}^{*}=5$, $h_{\mathrm{A}}^{*}=h_{\mathrm{B}}^{*}=10^{-4}$. From Table I we determine $y_{\mathrm{A}}=5$,

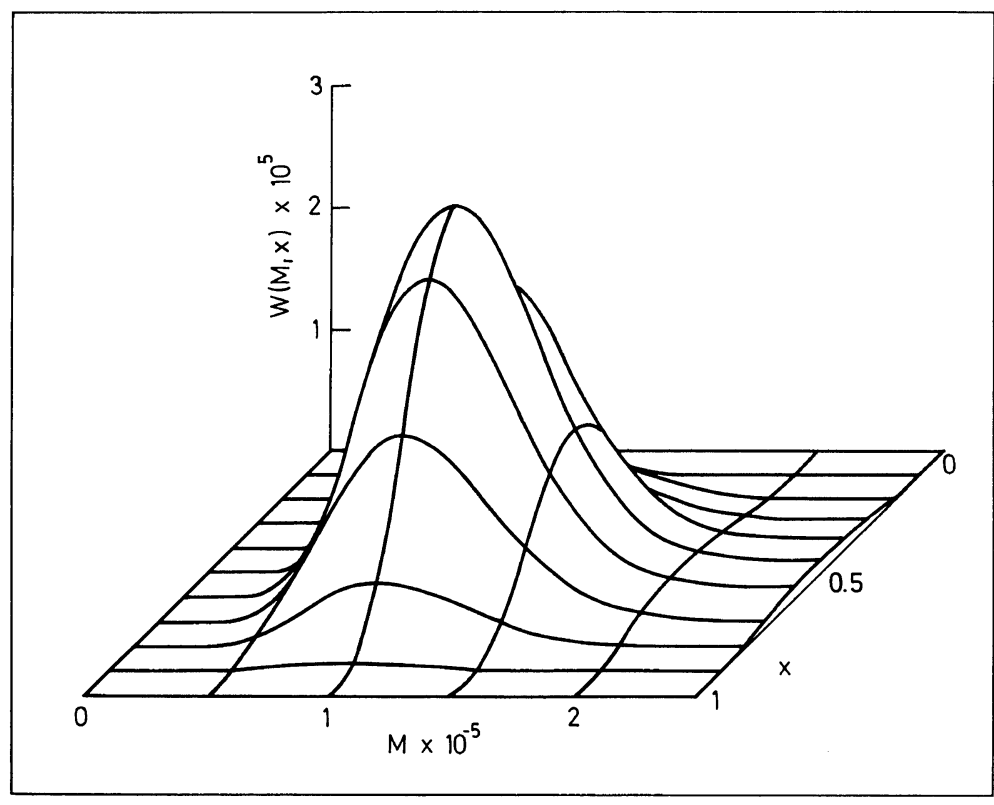

3 (a) 


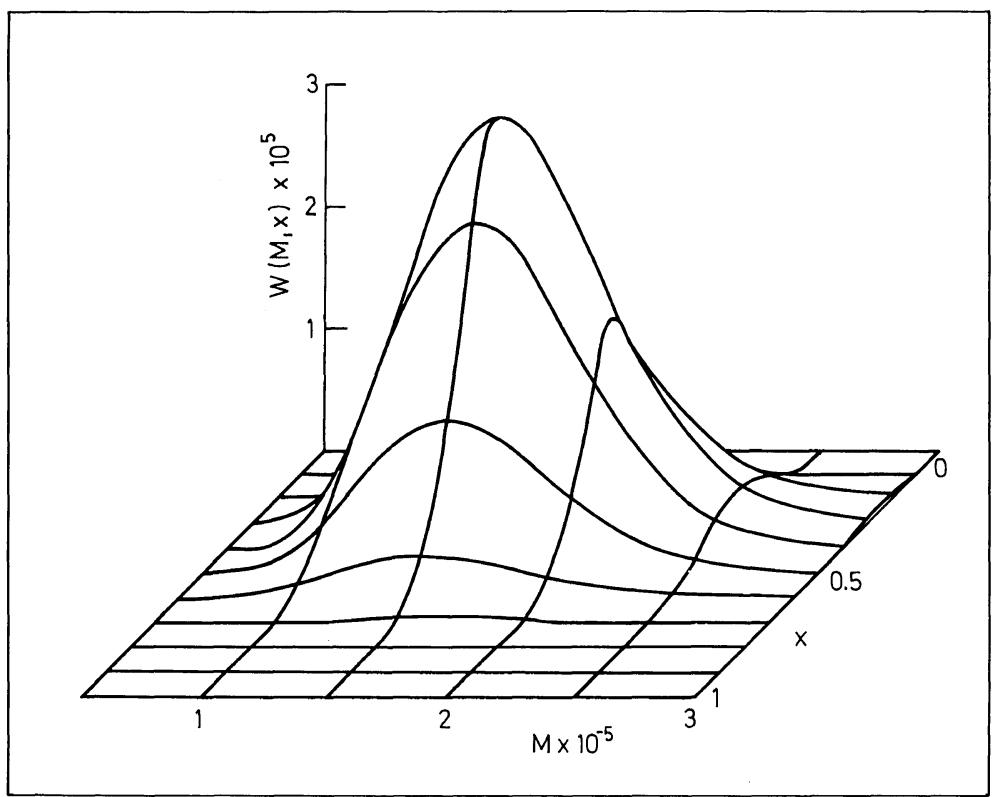

3 (b)

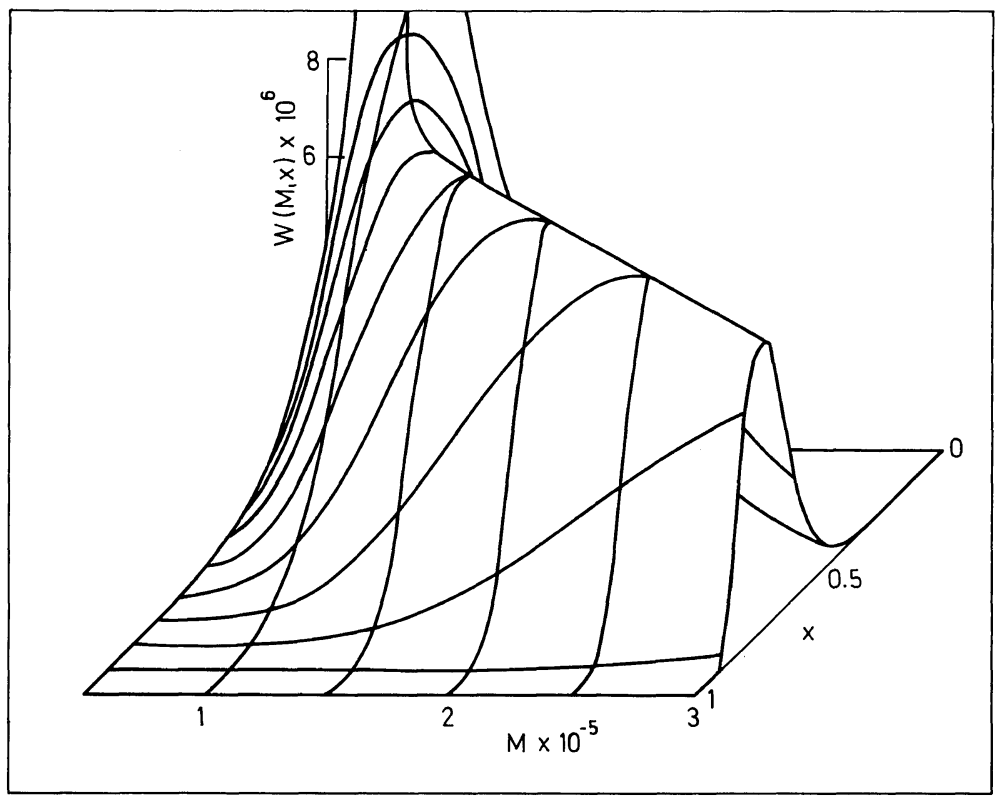

3 (c)

Figure 3. Two-dimensional differential weight distribution function $W(M, x)$ of molecular weights, $M$, and chemical composition, $x$, for (a) diblock AB and (b) triblock BAB (in both cases, $M_{n \mathrm{~A}}^{*}=M_{n \mathrm{~B}}^{*}=50,000$, $M_{w \mathrm{~A}}^{*} / M_{n \mathrm{~A}}^{*}=M_{w \mathrm{~B}}^{*} / M_{n \mathrm{~B}}^{*}=1.2$ ), and (c) triblock BAB where $M_{n \mathrm{~A}}^{*}=70,000, M_{n \mathrm{~B}}^{*}=40,000, M_{w \mathrm{~A}}^{*} / M_{n \mathrm{~A}}^{*}=3, M_{w \mathrm{~B}}^{*} /$ $M_{n \mathrm{~B}}^{*}=1.2$. 


\section{Compositional Distributions of Copolymers}

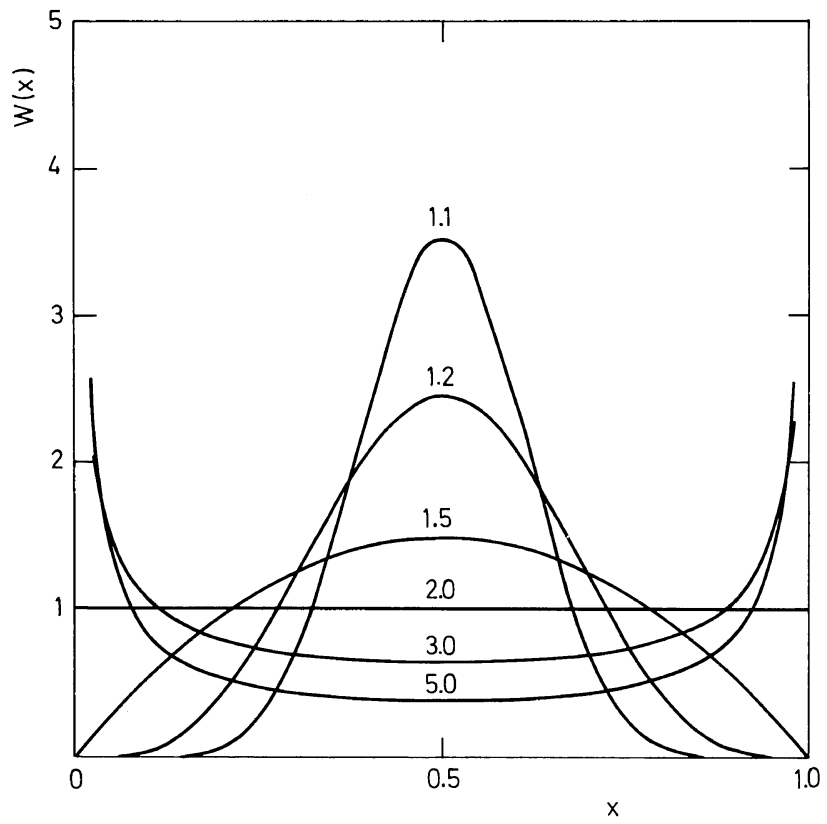

4 (a)

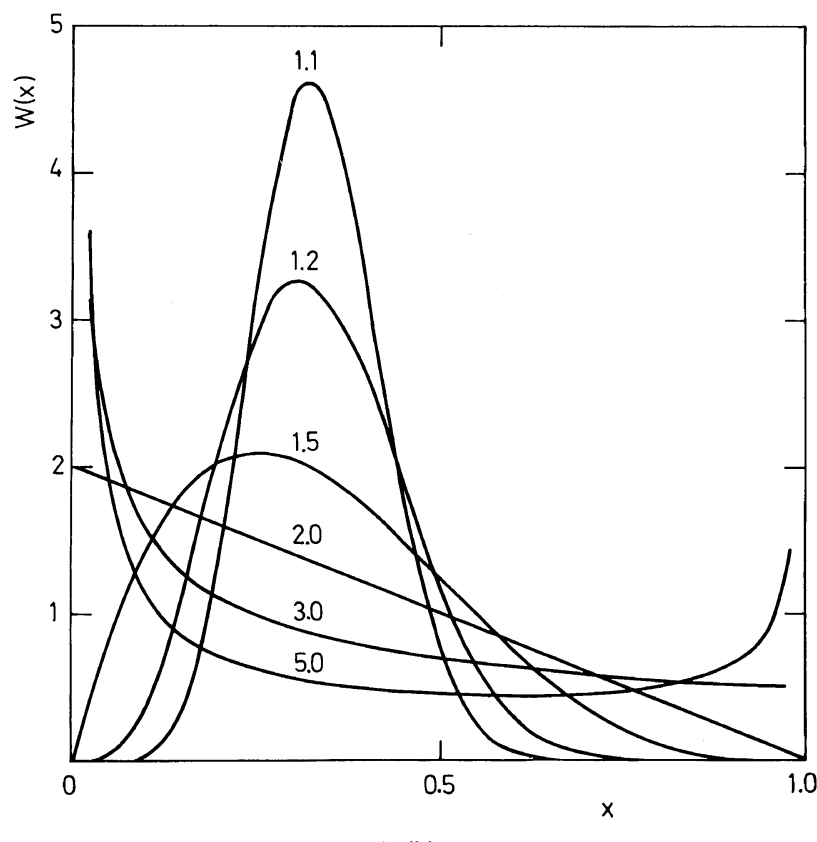

4 (b)

Figure 4. Marginal differential weight distribution functions $W(x)$ of chemical composition, $x$, of (a) model diblock $\mathrm{AB}$, and (b) model triblock BAB formed by joining blocks A and B with the same $\Gamma$ distribution of molecular weights having polydispersity $M_{w}^{*} / M_{n}^{*}$ indicated for individual curves. 
$y_{\mathrm{B}}=5$ for the diblock and $y_{\mathrm{B}}=10$ for the triblock, $h_{\mathrm{A}}=h_{\mathrm{A}}^{*}, h_{\mathrm{B}}=h_{\mathrm{B}}^{*}$. The two-dimensional distribution functions $W(M, x)$ have for this case the form of eq 33 (Figures $3 a, b$ )

$$
\begin{array}{ll}
W(M, x)=\Gamma\left(M ; 11,10^{-4}\right) \mathrm{B}(x ; 5,5) & \text { (diblock) } \\
W(M, x)=\Gamma\left(M, 16,10^{-4}\right) \mathrm{B}(x ; 5,10) & \text { (triblock) }
\end{array}
$$

Figure $3 \mathrm{c}$ shows a more general case of such distribution function.

The marginal distribution functions of chemical composition for a model diblock ${ }^{1}$ and triblock are relatively broad (Figure 4), even if both precursors have a narrow molecular weight distribution. This means, in practice, that the chemical heterogeneity of block copolymers cannot, as a rule, be neglected. The case where for both precursors $M_{w}^{*} / M_{n}^{*}=2$ is of interest (the so-called most probable distribution). The chemical composition of the diblock has then a uniform distribution (Figure 4a), i.e., copolymer macromolecules with any composition are present in identical relative amounts. Under the same conditions, the distribution of the triblock copolymer is triangular (Figure 4b). If the polydispersity indices of the precursors $M_{w}^{*} / M_{n}^{*}>2$, the distribution functions of chemical composition are concave, and the content of macromolecules having a composition close to that of homopolymers increases compared with the preceding cases.

Such a trend could be expected. For instance, with a diblock copolymer, because of the broad molecular weight distributions of the precursors, the probability of joining of two macromolecules with the same molecular weight (i.e., the occurrence of the composition $x=0.5$ ) is obviously low.

The distribution functions of chemical composition of graft copolymers can be represented in a similar way. Figure 5 shows such functions for a graft copolymer consisting of one backbone to which $m$ grafts have been attached; the grafts are attached according to model II. In this case too, one can see a strong effect of the initial polydispersity of parent polymers on the final form of the distribution functions.

A real graft copolymer consists of a mixture of more species, and its distribution function of chemical composition is a weighted sum of the distribution functions of these species, some of which are depicted in Figure 5.

\section{Parameters of Chemical Heterogeneity}

A preliminary view of the magnitude of the parameters of chemical heterogeneity of both block

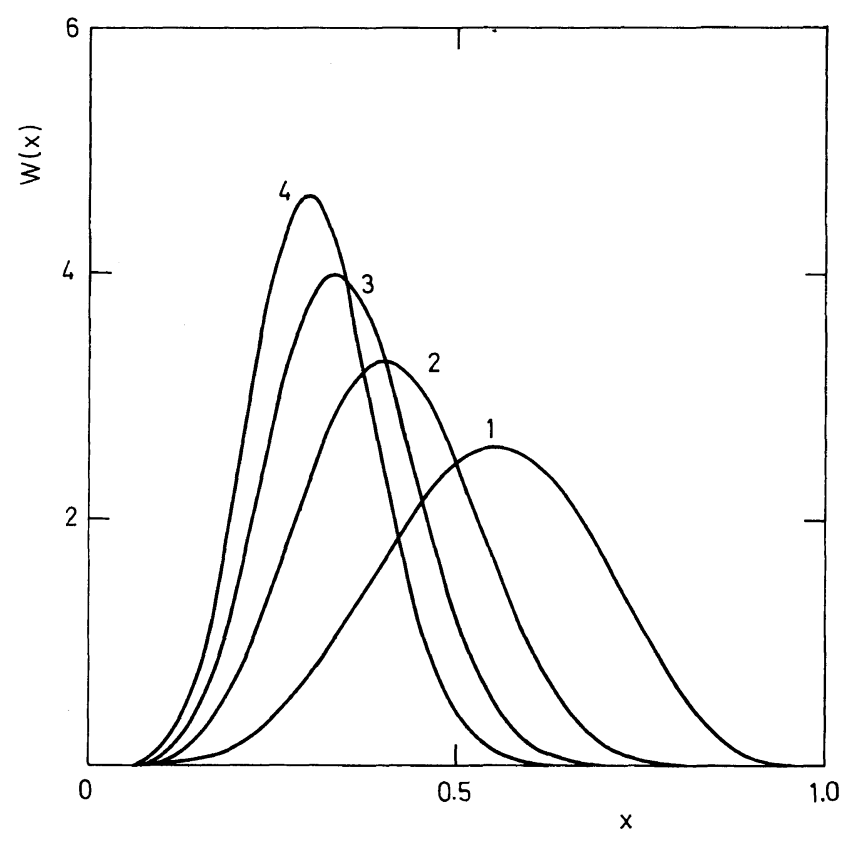

5 (a) 


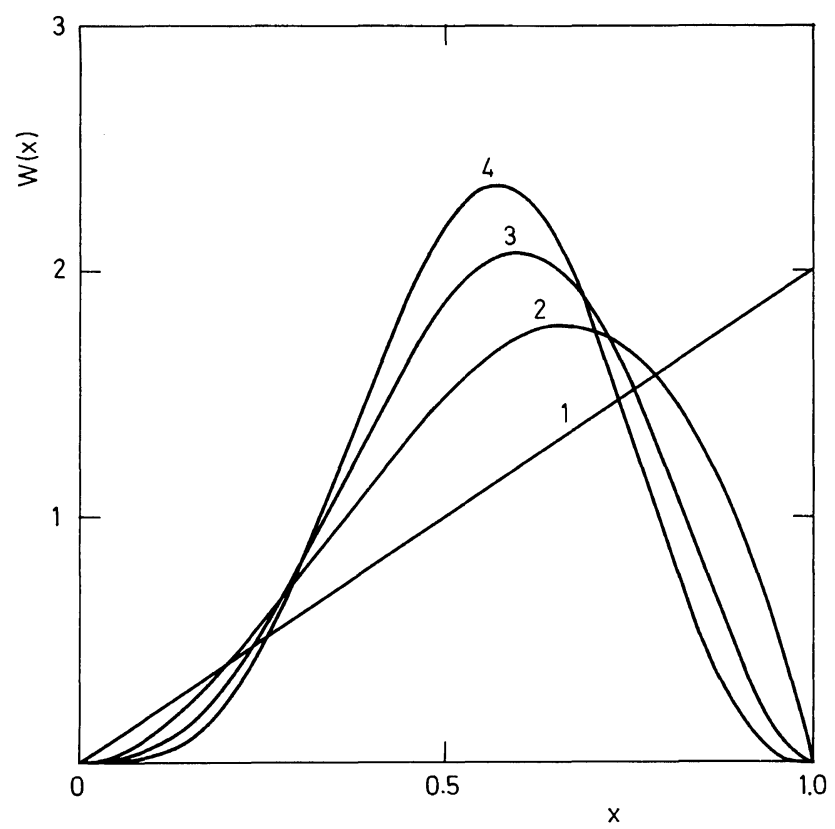

5 (b)

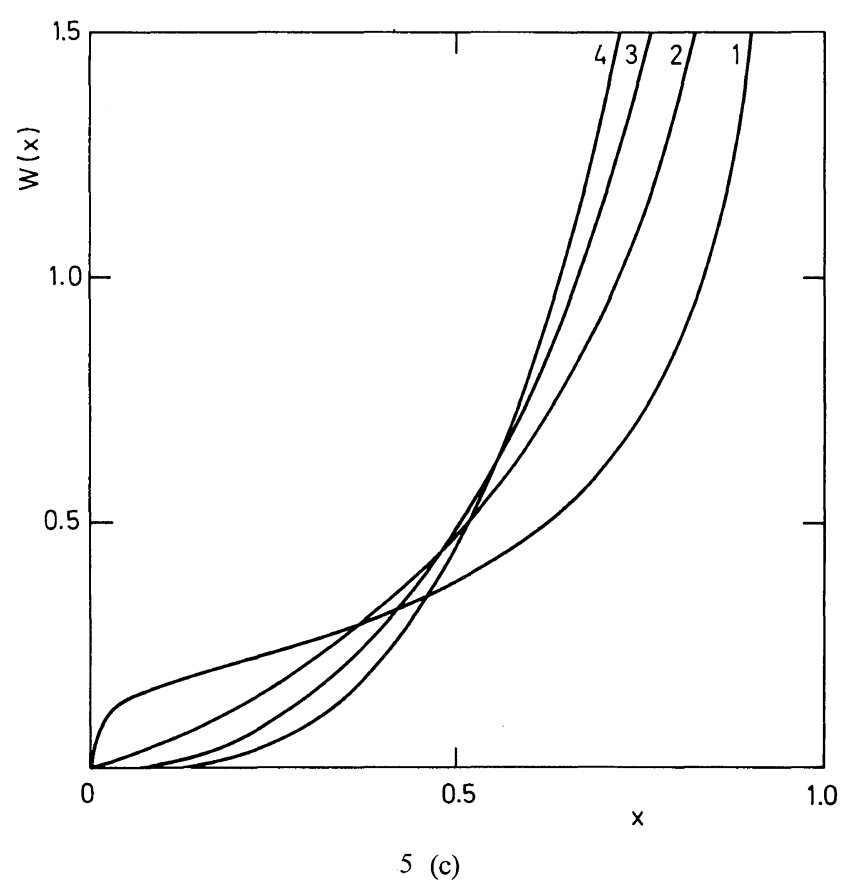

Figure 5. Marginal differential weight distribution function $W(x)$ of chemical composition, $x$, of graft copolymer bearing on one backbone of type A $m$ grafts of type B. Number of grafts $m$ is given for individual curves. Both blocks A and B have the same $\Gamma$-distribution of molecular weights, $M_{w}^{*} / M_{n}^{*}=(\mathrm{a})$ 1.2 , (b) 2 , and (c) 5 . 
J. Stejskal and P. Kratochvíl

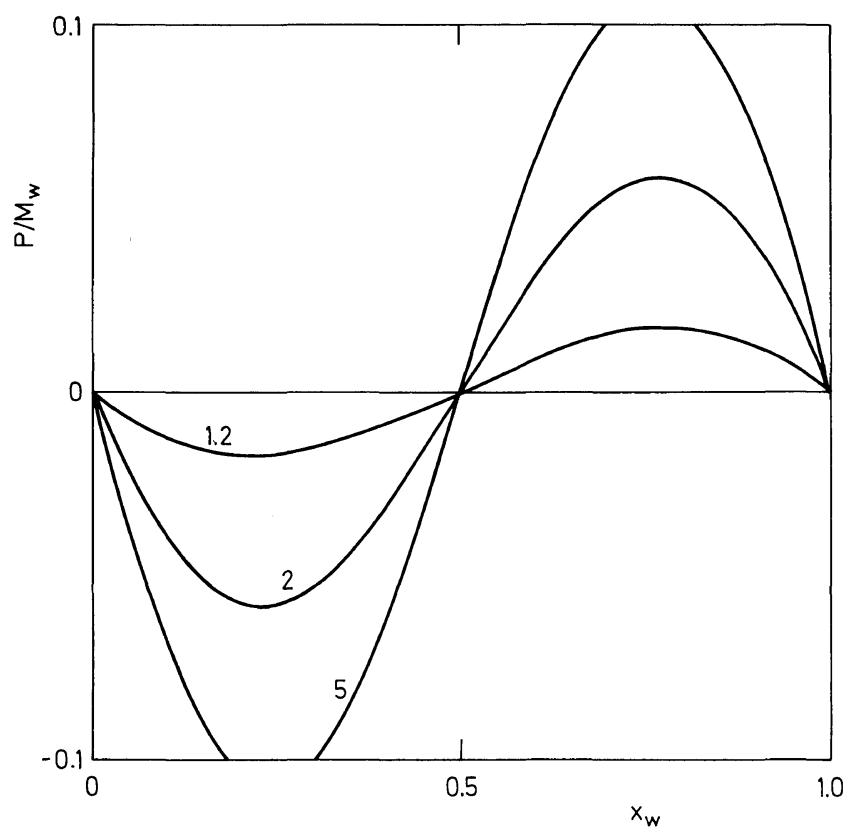

$6($ a)

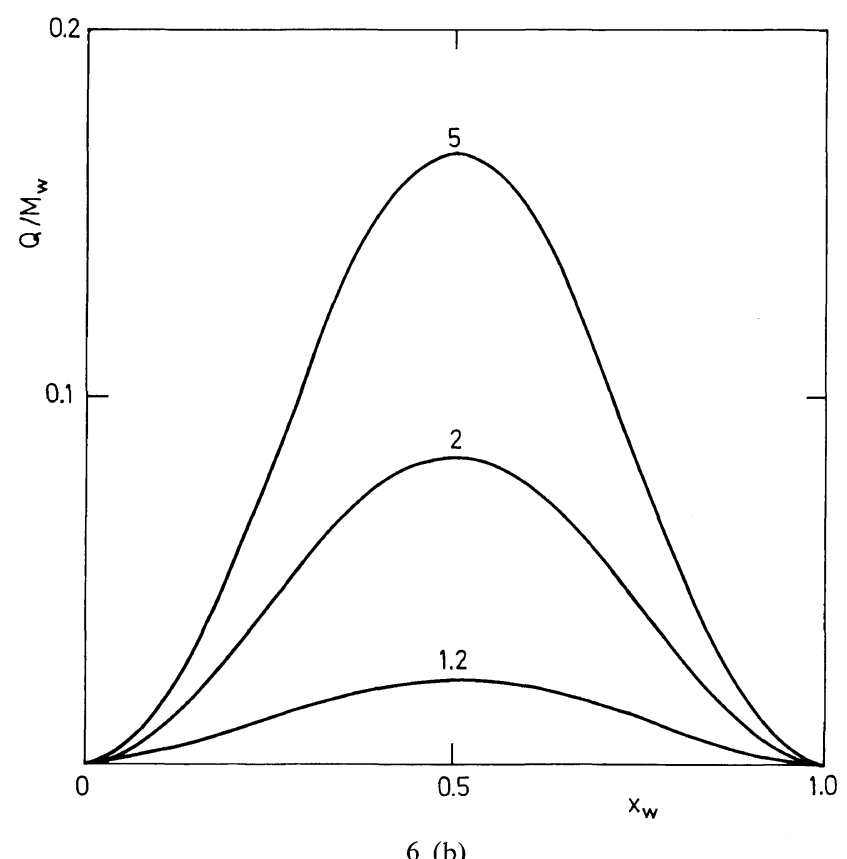

Figure 6. Parameters of chemical heterogeneity (a) $P / M_{w}$, and (b) $Q / M_{w}$ of copolymer whose parts A and B have polydispersity $M_{w} / M_{n}=1.2,2,5$ (assumed to be the same for both parts of the copolymer) depending on average chemical composition of copolymer, $x_{w}$. 
and graft copolymers can be obtained from Figure 6 , where these parameters are graphically represented according to eq 36 and 37 as a function of the chemical composition of the copolymer for various polydispersities of copolymer parts, $M_{w} / M_{n}$ (assumed the same for both copolymer parts).

The following trends can be stated:

(a) The absolute value of the parameters $P / M_{w}$ and $Q / M_{w}$ increases with increasing polydispersity of molecular weight of the individual parts of the copolymer. The more precursor molecules participate in the build-up of a copolymer macromolecule, the lower is the polydispersity of copolymer parts (Table I, eq 8b), and consequently, the lower are the parameters of chemical heterogeneity of such copolymer.

(b) If the number average molecular weights of the individual copolymer parts, each of which may consist of more blocks, are the same $\left(x_{w}=0.5\right)$, and if they have the same polydispersity, then $P / M_{w}=0$ while $Q / M_{w}$ assumes its maximum for the given polydispersity $M_{w} / M_{n}$.

(c) Both parameters, $\left|P / M_{w}\right|$ and $Q / M_{w}$, are high, exceeding by several orders of magnitude values common with statistical copolymers prepared up to medium and high conversions. ${ }^{15}$ In many cases, the parameters of chemical heterogeneity are so high that they can with sufficient accuracy be determined by light scattering, ${ }^{16}$ or the chemical heterogeneity of copolymers can be characterized by another method. ${ }^{15}$

Comparison of Models of Formation of Copolymers

Figure 7 shows how the form of the distribution function of chemical composition is affected by the model chosen, i.e., by the mechanism of formation of the copolymer. As expected, the differences are relatively small if the molecular weight distributions of the precursors are very narrow and become increasingly pronounced with broadening polydispersity of the latter. In this case, too, several rather general conclusions can be drawn:

(a) The distribution functions of chemical composition for model I are U-shaped (convex) for $y_{\mathrm{A}}$, $y_{\mathrm{B}}<1$ and $\Pi$-shaped for $y_{\mathrm{A}}, y_{\mathrm{B}}>1$, or have a more complicated form for other combinations of these parameters ( $c f .$, e.g., Figure 9). On the other hand, as follows from Table I, $y_{\mathrm{A}}, y_{\mathrm{B}}>1$ always hold for model III, i.e., the distribution functions for model III are always $\Pi$-shaped. For model II, we always have $y_{\mathrm{A}}>1$, i.e., the distributions are never $\mathrm{U}$ shaped.

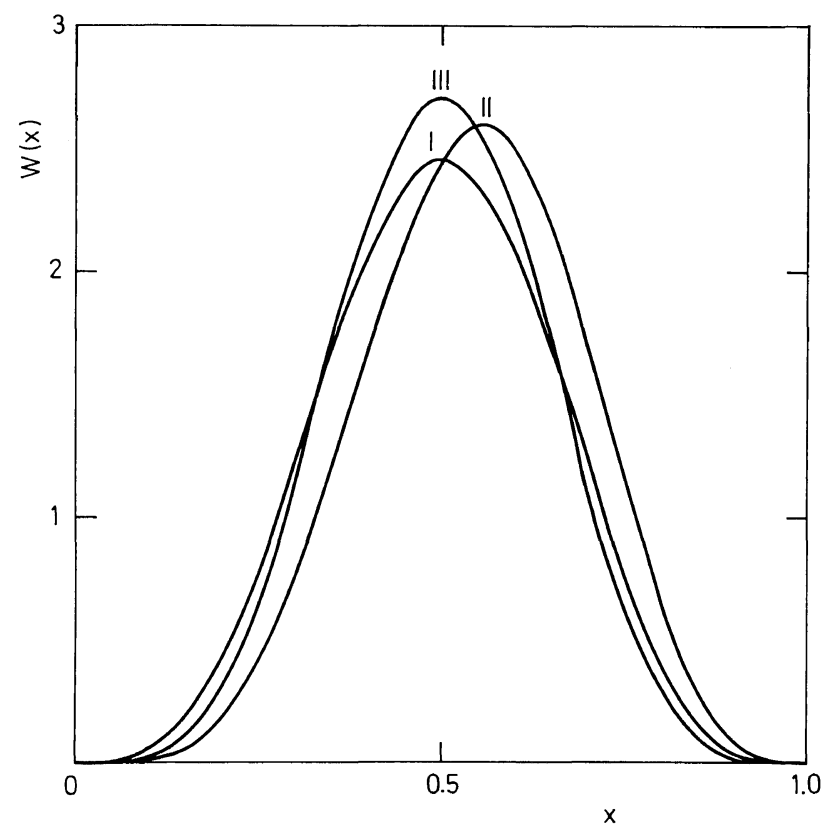

7 (a) 


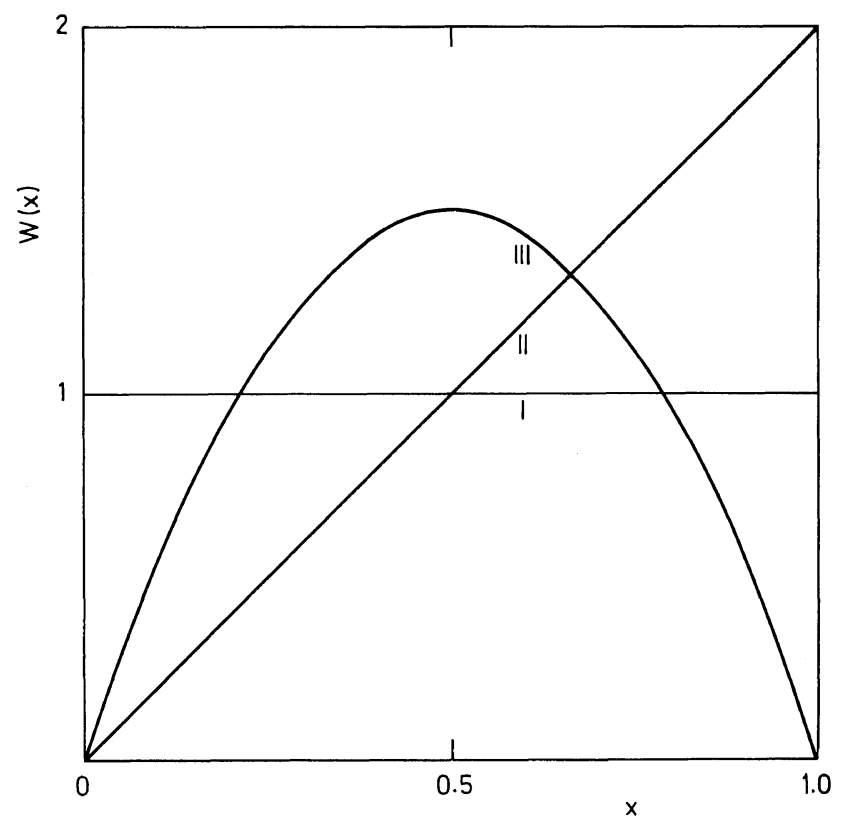

7 (b)

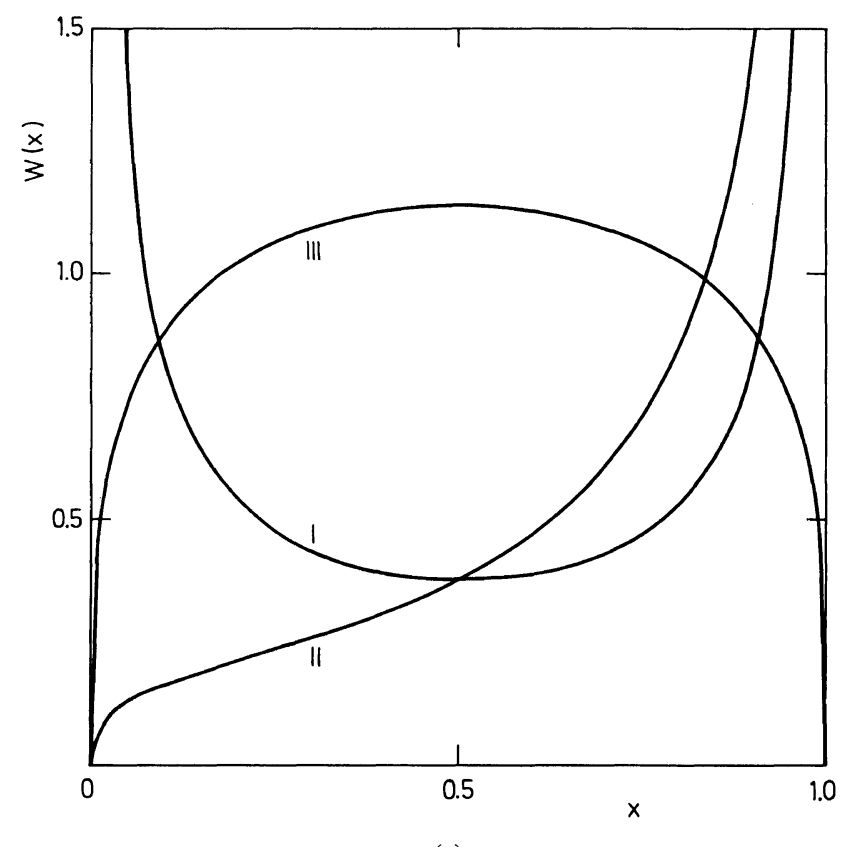

7 (c)

Figure 7. Comparison of marginal differential weight distribution functions $W(x)$ of chemical composition, $x$, for copolymer obtained by joining one block A and one block B with the same $\Gamma$-distribution of molecular weights having $M_{w}^{*} / M_{n}^{*}=$ (a) 1.2 , (b) 2 , and (c) 5 for individual models I through III. 
(b) Model III always gives narrower distributions of chemical composition than model I.

(c) For a high polydispersity of precursors, $y_{\mathrm{A}}$, $y_{\mathrm{B}} \rightarrow 0$, the distribution of chemical composition for model III approaches a uniform distribution.

These conclusions obviously hold only on the assumption that the precursors have $\Gamma$-distributions of molecular weights. In the discussion below, it is shown, however, that the type of molecular weight distribution of the precursors does not affect the conclusions to any essential degree.

\section{Approximation of Equal Blocks}

Quite often, the distribution function of molecular weights of copolymer parts cannot be expressed in an analytical form, especially if the distribution functions of molecular weights of precursors have a rather complicated character. For this reason, the so-called approximation of equal blocks is sometimes introduced in the literature. ${ }^{1,4}$ It is assumed that all precursor blocks of the A or B type which form a given copolymer macromolecule have the same molecular weight, but have different weights in different macromolecules in ac- cord with the assumed type of molecular weight distribution of the precursors. This means that, e.g., the molecular weight of part $\mathrm{A}$ of the copolymer molecule composed of $a$ blocks, $M_{\mathrm{A}}=$ $M_{\mathrm{A} 1}^{*}+M_{\mathrm{A} 2}^{*}+\cdots+M_{\mathrm{A} a}^{*}$, is approximated by the molecular weight $M_{\mathrm{A}}=a M_{\mathrm{A}}^{*}$, which in terms of the distribution functions can be written as (cf. eq 10)

$$
N_{\mathrm{A}}\left(M_{\mathrm{A}} \mid a\right) \approx N_{\mathrm{A}}^{*}\left(a M_{\mathrm{A}}^{*}\right)
$$

If the precursor blocks have the $\Gamma$-distribution of molecular weights, then ( $c f .$, e.g., eq 12a)

$$
N_{\mathrm{A}}\left(M_{\mathrm{A}} \mid a\right) \approx \Gamma\left(M_{\mathrm{A}} ; y_{\mathrm{A}}^{*}, h_{\mathrm{A}}^{*} / a\right)
$$

A similar relation holds for the precursor blocks B. Further equations which apply to the distribution of chemical composition of the copolymer are the same as in the cases already discussed, only relations between the parameters $y_{\mathrm{A}}$ and $y_{\mathrm{A}}^{*}, h_{\mathrm{A}}$ and $h_{\mathrm{A}}^{*}$ are different, as summarized in Table I.

The above approximation does not respect the important fact that by interlinking the blocks the molecular weight distribution of the copolymer part thus obtained becomes narrower. It can be seen in

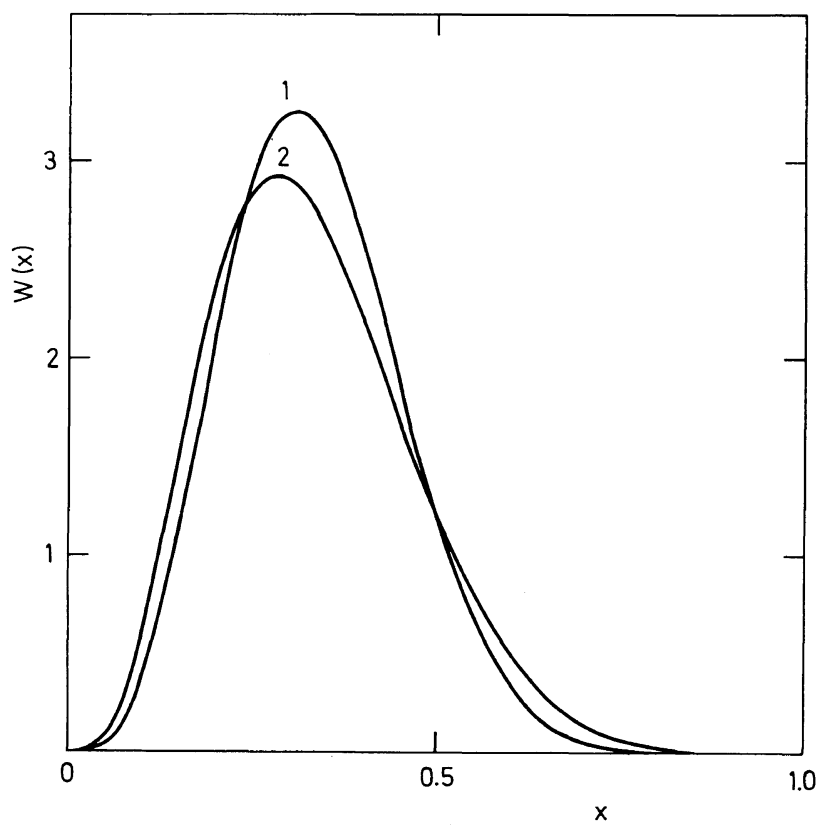

Figure 8. Comparison of marginal differential weight distribution functions $W(x)$ of chemical composition, $x$, of triblock BAB (1) and of the same function calculated with approximation of equal blocks B in given copolymer macromolecule (2). For both precursor block identical $\Gamma$-distribution of molecular weights is assumed, $M_{w}^{*} / M_{n}^{*}=1.2$. 
eq 43 that the parameter $y_{\mathrm{A}}=y_{\mathrm{A}}^{*}$ does not depend on the number $a$ of the joined blocks, and thus that, according to eq 8 , the ratio $M_{w \mathrm{~A}} / M_{n \mathrm{~A}}$ is also independent of $a$.

The approximation specified by eq 42 holds exactly for a diblock or for a graft copolymer with one graft. Already in a triblock, the differences between calculated distribution functions cannot be neglected (Figure 8), and the difference increases further with increasing number of the joined blocks. The use of this approximation predicts always a broader distribution of chemical composition, and hence also a higher chemical heterogeneity of the copolymer compared to a more rigorous calculation without such approximation. Consequently, the approximation is not advised wherever statistical analysis or computing technique make possible calculation without such simplifying assumptions.

\section{Number and Weight Distributions of Chemical Composition}

For chemically heterogeneous copolymers, two basic types of the distribution functions of chemical composition can be distinguished, viz., the number,
$N(x)$, and the weight, $W(x)$, functions which give the number and weight fractions, respectively, of macromolecules having the given composition, $x$. This is an analogy of the common number and weight distribution functions of molecular weights.

If the distributions of molecular weights and of chemical compositions of the copolymer are mutually independent, i.e., if a two-dimensional distribution function can be written as a product of the corresponding marginal functions, e.g., $N(M, x)=$ $N(M) N(x)$, it can be derived from eq 2 and 3 that $N(x)$ and $W(x)$ are identical ( $c f$. discussion of eq 33), because

$$
\begin{aligned}
W(x) & =\int_{M} W(M, x) \mathrm{d} M=M_{n}^{-1} \int_{M} M N(M, x) \mathrm{d} M \\
& =N(x) M_{n}^{-1} \int_{M} M N(M) \mathrm{d} M=N(x)
\end{aligned}
$$

In a general case, $N(x)$ and $W(x)$ differ from each other (Figure 9, cf. also Figure 3c). Hence, the number and weight averages of chemical composition, $x_{\mathrm{n}}$, defined by eq $5 \mathrm{a}$, and $x_{w}$ are also different (for the case illustrated in Figure 9, $x_{\mathrm{n}}=0.33$ and $\left.x_{w}=0.47\right)$. Thus, in principle, the ratio

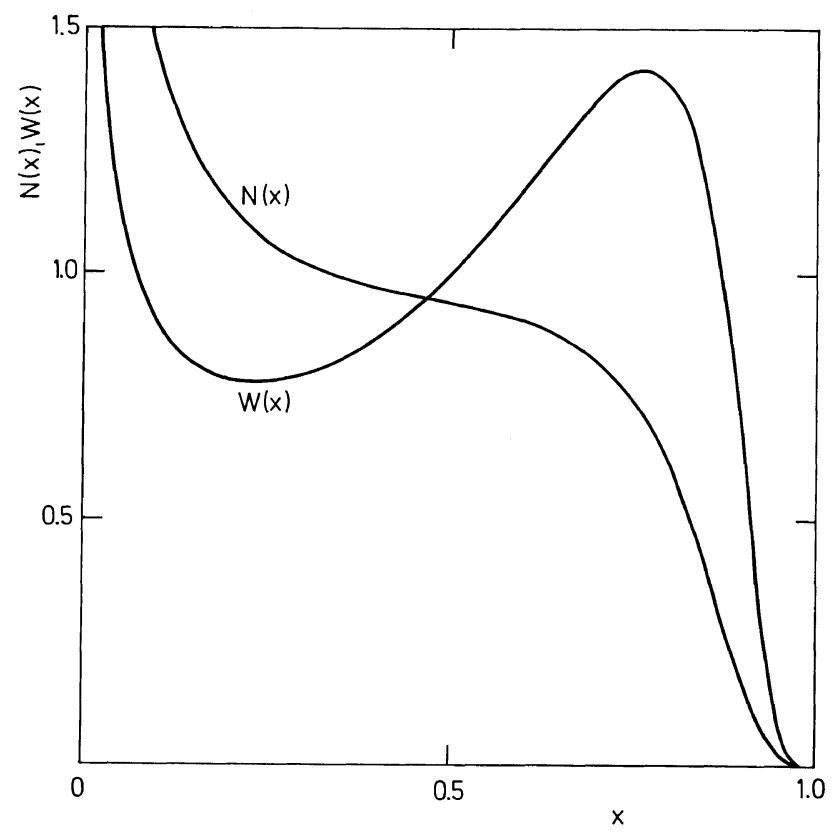

Figure 9. Comparison of marginal differential number and weight distribution functions, $N(x)$ and $W(x)$, of chemical composition, $x$, for the model triblock BAB. $\Gamma$-distribution, $M_{n \mathrm{~A}}^{*}=70,000, M_{n \mathrm{~B}}^{*}=$ $40,000, M_{w \mathrm{~A}}^{*} / M_{n \mathrm{~A}}^{*}=3, M_{w \mathrm{~B}}^{*} / M_{n \mathrm{~B}}^{*}=1.2$. 
$x_{\mathrm{w}} / x_{n}$ could serve as another parameter of chemical heterogeneity having a meaning similar to that of parameter $P$.

All experimental methods of determination of the chemical composition of copolymers give the weight average value, $x_{w}$; we are not aware of the existence of any procedure in use which would yield the number average value, $x_{n}$. In principle, however, methods leading to its determination could be suggested. Thus, e.g., if each macromolecule caried one certain characteristic group (fluorescence, isotope, or other label), it would become possible, after the fractionation of the sample according to chemical composition, to determine not only the weight, but also the number of macromolecules having the given composition in each fraction, and thus to derive the number distribution of chemical composition and its average.

For the sake of completeness, it should be mentioned that all the distribution functions discussed here can have their variable chemical composition expressed not only through the weight fraction, $x$, of one of the monomer units, but also through the mole fractions, $\xi$, of these units. For all distribution functions, both these ways of expression are related by

$$
W^{\prime}(\xi)=\frac{t}{[\xi(t-1)+1]^{2}} W\left[\frac{\xi t}{\xi(t-1)+1}\right]
$$

where $t=M_{\mathrm{OA}} / M_{\mathrm{OB}}$ is the ratio of molecular weights of the monomer units, and $x=\xi t /[\xi(t-1)+1]$.

\section{Comparison of Various Types of Molecular Weight Distributions}

It has been assumed throughout this study that the molecular weights of precursors (parent polymers) have the $\Gamma$-distribution. It is undoubtedly a question of principle whether and how the conclusions would change if the character of the distribution is different. We have, therefore, examined also some special cases where both parts A and B of the copolymer have an identical Tung or logarithmic-normal distribution.

(a) Tung distribution. Let both the parts $\mathrm{A}$ and $\mathrm{B}$ of the copolymer (or the precursors A and B, if the copolymer is a diblock) have an identical Tung number molecular weight distribution ${ }^{17}$

$$
N(M)=T(M ; y, h)=y h M^{y-1} \exp \left(-h M^{y}\right)
$$

where the positive parameters of the distribution function, $y$ and $h$, are related with the molecular weight averages by

$$
\begin{aligned}
& M_{n}=h^{-1 / y} \Gamma\left(1+\frac{1}{y}\right) \\
& M_{w} / M_{n}=\Gamma\left(1+\frac{2}{y}\right) / \Gamma^{2}\left(1+\frac{1}{y}\right)
\end{aligned}
$$

Then, the two-dimensional distribution function of molecular weights and chemical composition has the form

$$
\begin{gathered}
W(M, x)=\frac{h^{(2+1 / y)} y^{2}}{2 \Gamma(1+1 / y)}[x(1-x)]^{y-1} M^{2 y} \\
\exp \left\{-\left[x^{y}+(1-x)^{y}\right] h M^{y}\right\}
\end{gathered}
$$

and the marginal number and weight distribution of chemical composition are given by

$$
\begin{aligned}
& N(x)=y \frac{[x(1-x)]^{y-1}}{\left[x^{y}+(1-x)^{y}\right]^{2}} \\
& W(x)=\frac{y+1}{2} \frac{[x(1-x)]^{y-1}}{\left[x^{y}+(1-x)^{y}\right]^{(2+1 / y)}}
\end{aligned}
$$

(b) Logarithmic-normal distribution. Similarly to the preceding case, let the parts $\mathrm{A}, \mathrm{B}$ of a copolymer have an identical logarithmic-normal molecular weight distribution

$$
\begin{aligned}
N(M) & =\operatorname{lo}\left(M ; y, h^{2}\right) \\
& =\frac{1}{\sqrt{2 \pi} h M} \exp \left[-\frac{1}{2 h^{2}} \ln ^{2} \frac{M}{M_{0}}\right]
\end{aligned}
$$

where the distribution parameters $y=\ln M_{0}$ and $h^{2}$ are related to the molecular weight averages by

$$
\begin{aligned}
& M_{n}=M_{0} \exp \left(h^{2} / 2\right) \\
& M_{w} / M_{n}=\exp \left(h^{2}\right)
\end{aligned}
$$

Then, the two-dimensional distribution function $W(M, x)$ has the form

$$
\begin{aligned}
& W(M, x)=\frac{1}{4 \pi h^{2} x(1-x) M_{0}} \\
& \exp \left[-\frac{1}{2 h^{2}}\left(\ln ^{2} \frac{x M}{M_{0}}+\ln ^{2} \frac{(1-x) M}{M_{0}}\right)-\frac{h^{2}}{2}\right]
\end{aligned}
$$

and the marginal distribution functions are given by 


$$
\begin{aligned}
N(x)= & \frac{1}{2 \sqrt{\pi} h x(1-x)} \exp \left(-\frac{1}{4 h^{2}} \ln ^{2} \frac{x}{1-x}\right) \\
W(x)= & \frac{1}{4 \sqrt{\pi} h x(1-x)^{2}} \\
& \exp \left[-\left(\frac{1}{2 h} \ln \frac{x}{1-x}+\frac{h}{2}\right)^{2}\right]
\end{aligned}
$$

It can be seen from eq 53a, among others, that the number distribution of $x /(1-x)$ is again logarithmic-normal. A more general case, when the logarithmic-normal molecular weight distributions of copolymer parts are not identical has been treated by Tanaka et al. ${ }^{11}$

By comparing the courses of the distribution functions of chemical composition calculated assuming different molecular weight distributions of the copolymer parts A and B and their different polydispersities (Figure 10), the following conclusions can be drawn:

(a) At a low polydispersity of the copolymer parts $\mathrm{A}$ and $\mathrm{B}$, the distributions of chemical composition are virtually independent of the type of molecular weight distribution (Figure 10a). This conclusion is also supported by the calculations performed by Tanaka et al. ${ }^{11}$ (b) If for both parts of the copolymer $M_{w} / M_{n}=$ 2 , an identical uniform distribution of chemical composition of the copolymer is obtained for both the Tung and the $\Gamma$-distribution. For the logarithmic-normal molecular weight distribution, the distribution of chemical composition is virtually uniform in the composition range between $x=0.3$ and 0.7 (Figure 10b).

(c) Even for broad molecular weight distributions, the character of the compositional distribution depends only very little on the type of the molecular weight distribution. The vertical displacement of curves in Figure $10 \mathrm{c}$ is mainly due to the different marginal behaviour of the distribution functions. In this case, the distributions of chemical composition calculated for the Tung and $\Gamma$-molecular weight distributions have at the boundaries of the interval of the composition $x$, the limit $+\infty$; function 1 for the $\Gamma$-distribution increases more quickly that function 2 for the Tung distribution (Figure 10c). Curve 3, which corresponds to the logarithmic-normal distribution, has both limits equal to zero; hence, in this case the distribution function of chemical composition exhibits a bimodal character with maxima localized at low $x$ values and $x$ values approaching unity. The bimodality of the same function with unpronounced

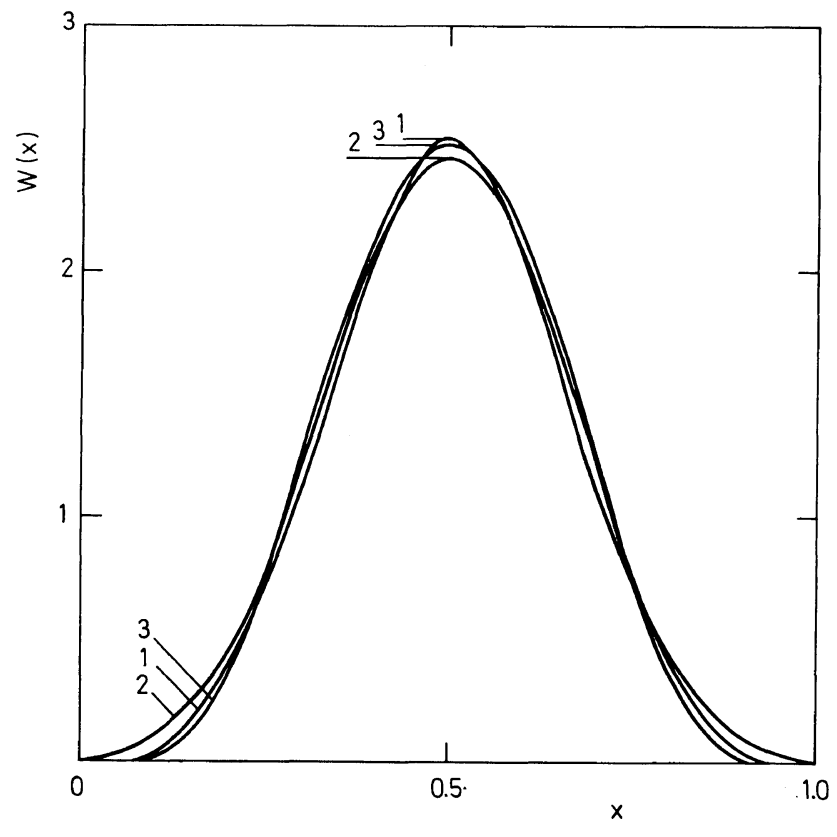

10 (a) 
Compositional Distributions of Copolymers

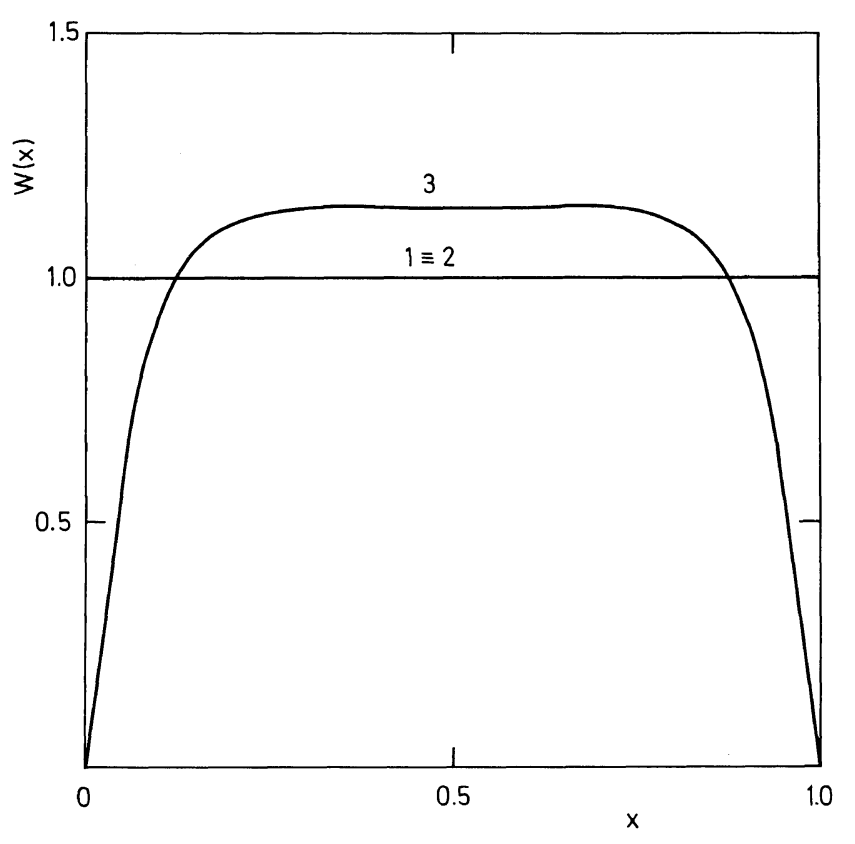

10 (b)

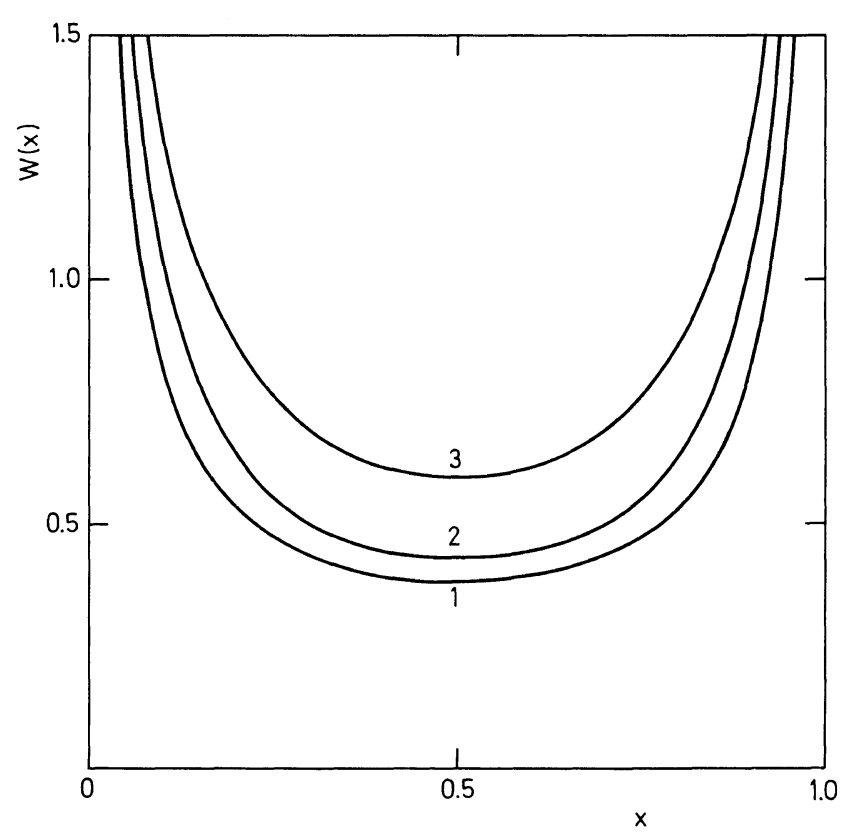

10 (c)

Figure 10. Marginal differential weight distribution functions $W(x)$ of chemical composition, $x$, of model diblock AB formed by combination of blocks A and B with identical molecular weight distribution: (1) $\Gamma$ (Schulz-Zimm), (2) Tung, and (3) logarithmic-normal of various width characterized by $M_{w}^{*} / M_{n}^{*}=$ (a) 1.2 , (b) 2, and (c) 5 . 
maxima for $x=0.33$ and $x=0.67$ is also slightly indicated in Figure 10b. The tendency to form copolymer macromolecules having a composition close to that of the homopolymers is most pronounced with the $\Gamma$-distribution and least pronounced with the logarithmic-normal distribution.

We believe, on the basis of this comparison, that the form of distributions of chemical composition is relatively little dependent on the type of the molecular weight distribution of the precursors, and, consequently, that approximation of a real molecular weight distribution of the precursors by the $\Gamma$ distribution is justified in the absolute majority of cases.

\section{APPENDIX A}

(A) Molecular Weight Distribution of the Copolymer Part Consisting of a Precursor Blocks We shall prove that, if the precursor blocks A have the $\Gamma$-distribution of molecular weights $N_{\mathrm{A}}^{*}\left(M_{\mathrm{A}}^{*}\right)=\Gamma\left(M_{\mathrm{A}}^{*} ; y_{\mathrm{A}}^{*}, h_{\mathrm{A}}^{*}\right)$ and the copolymer part containing $a-1$ joined blocks similarly has the molecular weight distribution

$$
\begin{aligned}
& N_{\mathrm{A}}\left(M_{\mathrm{A}}-M_{\mathrm{A} a}^{*} \mid a-1\right) \\
& \quad=\Gamma\left(M_{\mathrm{A}}-M_{\mathrm{A} a}^{*} ;(a-1) y_{\mathrm{A}}^{*}, h_{\mathrm{A}}^{*}\right)
\end{aligned}
$$

then the distribution of molecules weights $M_{\mathrm{A}}$ in a system containing $a$ joined blocks $\mathrm{A}$ is described by the distribution function $N_{\mathrm{A}}\left(M_{\mathrm{A}} \mid a\right)=\Gamma\left(M_{\mathrm{A}}\right.$; $a y_{\mathrm{A}}^{*}, h_{\mathrm{A}}^{*}$.

By substituting the $\Gamma$-distribution functions into eq 10 , we obtain

$$
\begin{aligned}
N_{\mathrm{A}}\left(M_{\mathrm{A}} \mid a\right)= & \int_{M_{\mathrm{A} a}^{*}<M_{\mathrm{A}}} \Gamma\left(M_{\mathrm{A} a}^{*} ; y_{\mathrm{A}}^{*}, h_{\mathrm{A}}^{*}\right) \\
& \times \Gamma\left(M_{\mathrm{A}}-M_{\mathrm{A} a}^{*} ;(a-1) y_{\mathrm{A}}^{*}, h_{\mathrm{A}}^{*}\right) \mathrm{d} M_{\mathrm{A} a}^{*}
\end{aligned}
$$

According to the definition of the $\Gamma$-distribution (eq 6) we have, then,

$$
\begin{aligned}
N_{\mathrm{A}}\left(M_{\mathrm{A}} \mid a\right) & \frac{h_{\mathrm{A}}^{*^{a y y_{\mathrm{A}}^{*}}}}{\Gamma\left(y_{\mathrm{A}}^{*}\right) \Gamma\left[(a-1) y_{\mathrm{A}}^{*}\right]} M_{\mathrm{A}}^{a y_{\mathrm{A}}^{*}-1} \exp \left(-h_{\mathrm{A}}^{*} M_{\mathrm{A}}\right) \\
& \int_{0}^{\infty}\left(1-\frac{M_{\mathrm{A} a}^{*}}{M_{\mathrm{A}}}\right)^{(a-1) y_{\mathrm{A}}^{*-1}}\left(\frac{M_{\mathrm{A} a}^{*}}{M_{\mathrm{A}}}\right)^{y_{\mathrm{A}}^{*-1}} \frac{\mathrm{d}_{M_{\mathrm{A} a}^{*}}^{*}}{M_{\mathrm{A}}}
\end{aligned}
$$

Substitution $\Xi=M_{\mathrm{Aa}}^{*} / M_{\mathrm{A}}$ yields

$$
\begin{aligned}
N_{\mathrm{A}}\left(M_{\mathrm{A}} \mid a\right) \\
=\frac{h_{\mathrm{A}}^{* a y_{\mathrm{A}}^{*}}}{\Gamma\left(y_{\mathrm{A}}^{*}\right) \Gamma\left[(a-1) y_{\mathrm{A}}^{*}\right]} M_{\mathrm{A}}^{a y_{\mathrm{A}}^{*}-1} \exp \left(-h_{\mathrm{A}}^{*} M_{\mathrm{A}}\right) \\
\quad \int_{0}^{1}(1-\Xi)^{(a-1) y_{\mathrm{A}}^{*-1}} \Xi^{y_{\mathrm{A}}^{*-1}} d \Xi
\end{aligned}
$$

On changing the integration boundaries we respect the requirement $M_{\mathrm{Aa}}^{*}<M_{\mathrm{A}}$, i.e., $\Xi<1$. The improper integral in (A-3) is $\Gamma\left[(a-1) y_{\mathrm{A}}^{*}\right] \Gamma\left(y_{\mathrm{A}}^{*}\right) /$ $\Gamma\left(a y_{\mathrm{A}}^{*}\right)$, and hence,

$$
N_{\mathrm{A}}\left(M_{\mathrm{A}} \mid a\right)=\frac{h_{\mathrm{A}}^{* a y_{\mathrm{A}}^{*}}}{\Gamma\left(a y_{\mathrm{A}}^{*}\right)} M_{\mathrm{A}}{ }^{a y_{\mathrm{A}^{*}}-1} \exp \left(-h_{\mathrm{A}}^{*} M_{\mathrm{A}}\right)(\mathrm{A}-4)
$$

By comparing with eq 6 , we obtain eq 12a,

$$
N_{\mathrm{A}}\left(M_{\mathrm{A}} \mid a\right)=\Gamma\left(M_{\mathrm{A}} ; a y_{\mathrm{A}}^{*}, h_{\mathrm{A}}^{*}\right)
$$

The resulting equation also follows directly from eq 33 , if the system of $a-1$ blocks is formally regarded as part B and the last joined block is regarded as part $\mathrm{A}$; then,

$$
W_{\mathrm{A}}\left(M_{\mathrm{A}} \mid a\right)=\Gamma\left(M_{\mathrm{A}} ; a y_{\mathrm{A}}^{*}+1, h_{\mathrm{A}}^{*}\right)
$$

and from eq 7 we again have eq $12 \mathrm{a}$.

\section{APPENDIX B}

\section{(B) Parameter $P$ of a Mixture of Chemically} Heterogeneous Copolymer Species

With regard to the general definition of the parameter $P$ given by eq $4 c$, it can be written for a mixture of chemically heterogeneous species $s$ that

$$
P_{\mathrm{C}}=\sum_{s} \iint_{x M}\left(x-x_{\mathrm{C}}\right) M W(M, x \mid s) W(s) \mathrm{d} M \mathrm{~d} x
$$

the parameter $P$ of the individual components of the mixture being

$$
P_{s}=\iint_{x M}\left(x-x_{s}\right) M W(M, x \mid s) \mathrm{d} M \mathrm{~d} x
$$

The weight average of $P_{s}$ is

$$
P_{s}^{(1)}=\sum_{s} P_{s} W(s)
$$

and the parameter $P$ of a hypothetical mixture whose individual components $s$ are chemically homogeneous is 


$$
P_{s}^{(2)}=\sum_{s}\left(x_{s}-x_{\mathrm{C}}\right) M_{s} W(s)
$$

After extension of the integral in eq B-1 and by changing the order of integration and summation, we obtain

$$
\begin{aligned}
P_{\mathrm{C}}= & \sum_{s} W(s) \iint_{x M}\left(x-x_{s}\right) M W(M, x \mid s) \mathrm{d} M \mathrm{~d} x \\
& +\sum_{s}\left(x_{s}-x_{\mathrm{C}}\right) W(s) \iint_{x M} M W(M, x \mid s) \mathrm{d} M \mathrm{~d} x
\end{aligned}
$$

and thus

$$
P_{\mathrm{C}}=\sum_{s} P_{s} W(s)+\sum_{s}\left(x_{s}-x_{\mathrm{C}}\right) W(s) M_{s}
$$

A comparison of the latter relation with eq B-2 through B-4 gives eq 4la. Derivation of an expression for the parameter $Q$ is quite similar.

\section{REFERENCES}

1. T. Kotaka, N. Donkai, and T. I. Min, Bull. Inst. Chem. Res., Kyoto Univ., 52, 332 (1974).

2. H. Inagaki, Adv. Polym. Sci., 24, 189 (1977).

3. T. Kotaka, T. Uda, T. Tanaka, and H. Inagaki,
Makromol. Chem., 176, 1273 (1975).

4. L. H. Tung and R. M. Wiley, J. Polym. Sci., Polym. Phys. Ed., 11, 1413 (1973).

5. T. Kotaka, Bull. Inst. Chem. Res., Kyoto Univ., 55, 135 (1977).

6. P. Cordier, J. Chim. Phys., 64, 439 (1967).

7. S. Teramachi, A. Hasegawa, S. Hasegawa, and T. Ishibe, Polym. J., 13, 319 (1981).

8. W. Bushuk and H. Benoît, Can. J. Chem., 36, 1616 (1958).

9. H. Benoit and D. Froelich, "Light Scattering from Polymer Solutions," M.B. Huglin, Ed., Academic Press, London, 1972, pp 467-501.

10. M. Leng and H. Benoît, J. Polym. Sci., 57, 263 (1962).

11. T. Tanaka, M. Omoto, N. Donkai, and H. Inagaki, J. Macromol. Sci., Phys., B17, 211 (1980).

12. P. Cordier, J. Chim. Phys., 64, 423 (1967).

13. J. Vorlíček and P. Kratochvíl, J. Polym. Sci., Polym. Phys. Ed., 11, 1251 (1973).

14. Y. Ikada and F. Horii, Makromol. Chem., 175, 227 (1974)

15. J. Stejskal and P. Kratochvíl, J. Appl. Polym. Sci., 22, 2925 (1978).

16. J. Vorlíček and P. Kratochvíl, J. Polym. Sci., Polym. Phys. Ed., 11, 855 (1973).

17. L. H. Tung, J. Polym. Sci., 20, 495 (1956). 